\title{
Real-Time Power Balancing in Electric Grids with Distributed Storage
}

\author{
Sun Sun, Student Member, IEEE, Min Dong, Senior Member, IEEE, and Ben Liang, Senior Member, IEEE
}

\begin{abstract}
Power balancing is crucial for the reliability of an electric power grid. In this paper, we consider an aggregator coordinating a group of distributed storage (DS) units to provide power balancing service to a power grid through charging or discharging. We present a real-time, distributed algorithm that enables the DS units to determine their own charging or discharging amounts. The algorithm accommodates a wide spectrum of vital system characteristics, including time-varying power imbalance amount and electricity price, finite battery size constraints, cost of using external energy sources, and battery degradation. We develop a modified Lyapunov optimization framework for real-time power balancing and provide a fast iterative method for distributed implementation. The two components interact through a novel cost cushion parameter that tunes the trade-off between system performance and convergence speed. We show analytically that the algorithm converges quickly and provides asymptotically optimal performance as the capacity of DS units increases. We further study through simulation the algorithm performance over a wide range of parameter values and demonstrate that it is highly competitive over a greedy alternative.
\end{abstract}

\section{INTRODUCTION}

Balancing power supply and demand, i.e., matching power generation and demand load continuously, is crucial for the reliability of an electric power grid. To achieve power balance, power grids schedule generation and load both in a large time scale (e.g., day-ahead or hour-ahead) based on the prediction of future supply and demand, and in a real-time scale (e.g., minutes or seconds) so as to clear power imbalance due to, for example, unavoidable prediction error [1]. For real-time scale power balancing, one of the most prevalent examples is frequency regulation, which operates every few seconds to maintain the frequency of a power grid at its nominal value, and is the most expensive ancillary service [2].

With the growing environmental concerns and the need to reduce greenhouse gas emissions, more and more renewable energy resources, such as wind and solar, are expected to be integrated into the future power grid. For example, the European Commission aims to include $20 \%$ renewable energy in the EU energy profile by 2020 [3], and California plans to achieve $33 \%$ of retail sales from renewable energy by 2020 [4]. However, as renewable generation is intermittent and difficult to predict, high penetration of renewable energy will create

Copyright (c) 2014 IEEE. Personal use of this material is permitted. However, permission to use this material for any other purposes must be obtained from the IEEE by sending a request to pubs-permissions@iee.org.

Sun Sun and Ben Liang are with the Department of Electrical and Computer Engineering, University of Toronto, Toronto, Canada (email: \{ssun, liang\}@comm.utoronto.ca).

Min Dong is with the Department of Electrical Computer and Software Engineering, University of Ontario Institute of Technology, Toronto, Canada (email: min.dong@uoit.ca). additional variations in the power system, and in particular new challenges to real-time power balancing.

To address the real-time power balancing problem, several intelligent algorithms have been proposed which aim at optimally scheduling either dispatchable generation on the supply side (e.g., [5] and [6]), or flexible load on the demand side (e.g., [7]). Complementary to these direct approaches, distributed storage (DS) units, such as batteries in electric vehicles and batteries deployed at renewable generators for regulating the rate of power supply, are potentially effective alternatives for real-time power balancing [8]. For example, experiments have revealed that an electric vehicle's power electronics and battery can well respond to frequent charging/discharging signals [9]. Thus, it is possible to exploit plugged-in electric vehicles to eliminate real-time power discrepancy.

There are many benefits in using DS units to balance power. Compared with supply side management using traditional generators, such as natural gas generators, which burn fossil fuels, DS units may be more environmentally friendly. Compared with scheduling power demands, intelligent charging/discharging of DS units may cause less inconvenience to users. Moreover, it is expected that there will be a large number of DS units in the near future. For example, based on the data published in [10], the cumulative U.S. plug-in vehicles sales had reached 180,000 in February 2014 since December 2010, and keep on rising. Additionally, with a significant growth of distributed photovoltaics, the number of battery-backed solar systems will increase accordingly [11]. Therefore, DS units will play important roles in the future power grid design and evolution, and in particular will create additional design choices for real-time power balancing.

However, the employment of DS units for real-time power balancing requires the participation of a large number of DS units, as the power imbalance amount in a power grid is in general much greater than the power capacity of an individual DS unit. For example, the typical power capacity of an electric vehicle is $5-20 \mathrm{~kW}$, in comparison with frequency regulation service requirement often on the order of megawatts. To coordinate participating DS units, it is often beneficial to have an aggregator. When serving power balancing, the aggregator could determine specific charging and discharging amounts for each DS unit. Nevertheless, since the DS units may belong to different owners, letting the aggregator fully control DS charging/discharging would override an owner's individual choice, thus potentially hampering its enthusiasm for participation [12]. Furthermore, the computational complexity of centralized control would dramatically increase as the number of participating DS units increases. An alternative approach, 
which is the focus of this paper, is to distribute the decision making to individual DS units.

In this paper, we consider a general problem of using DS units to provide real-time power balancing service for an electric power grid. We aim at offering both an optimal schedule of charging and discharging for each DS unit, and a fast distributed algorithm for its implementation. The proposed algorithm leverages the flexible charging and discharging capability of each DS unit and the bidirectional communication envisioned in the future power grid. Specifically, we consider an aggregator-DS system, in which the aggregator coordinates a large number of DS units exclusively serving power balancing. The aggregator is assumed to be regulated and non profit-driven, which can represent a government-funded party that encourages participation of DS units. The aggregator aims to minimize the long-term system cost, including its own cost and each DS unit's cost, in the presence of uncertain power imbalance amount and electricity price. Meanwhile, it has to respect each DS unit's battery capacity constraint as well as the degradation cost constraint associated with charging and discharging. This leads to a large-scale stochastic optimization problem. The problem is particularly challenging in two ways. First, in terms of real-time design, the dynamic system state and the finite battery size constraints complicate the joint decision making over multiple time instances. Second, in terms of distributed implementation of scheduling the DS units' charging and discharging amounts, the decision of each DS unit is intrinsically coupled with those of the others due to the system-wide objective, which hinders the development of a decentralized solution.

To tackle these two difficulties, we first use a modified Lyapunov optimization technique [13] to transform the original long-term objective into real-time sub-problems that respect the battery size constraints. Then, we employ Lagrange dual decomposition [14] and adapt a fast iterative shrinkagethresholding algorithm (FISTA) [15] to distributively solve the real-time sub-problems. We propose a novel cost cushion parameter, which integrates the aforementioned two components into a unified distributed optimization algorithm. The parameter is also designed to tune the trade-off between system performance and convergence speed. The proposed algorithm does not require any knowledge of the system statistics. We show analytically that the algorithm converges quickly and guarantees optimal performance asymptotically as each DS unit capacity increases. Through simulation studies, we characterize the performance of the proposed algorithm over a wide range of parameter values and demonstrate that it significantly outperforms a greedy alternative.

The remainder of this paper is organized as follows. The related works are summarized in Section II. In Section III, we describe the system model and formulate the power balancing problem for an aggregator-DS system. In Section IV, we decompose the original problem into real-time sub-problems, and in Section V, we provide a distributed solution to the realtime sub-problems and study its convergence performance. In Section VI, the overall algorithm is summarized and its optimality properties are evaluated. Simulations are presented in Section VII, and a discussion on dynamic DS units is given
TABLE I

List of MAIN SyMbols (IN THE ORDER OF APPEARANCE)

\begin{tabular}{|l|l|}
\hline$g_{t}$ & energy imbalance signal at time slot $t$ \\
\hline$g_{\text {max }}$ & maximum value of the energy imbalance signal \\
\hline $\mathbf{1}_{s, t}$ & indicator of energy surplus at time slot $t$ \\
\hline $\mathbf{1}_{d, t}$ & indicator of energy deficit at time slot $t$ \\
\hline$x_{i, t}$ & charging amount of the $i$-th DS during time slot $t$ \\
\hline$y_{i, t}$ & discharging amount of the $i$-th DS during time slot $t$ \\
\hline$r_{i, \text { max }}$ & maximum allowed charging/discharging amount of the $i$-th DS \\
\hline$s_{i, t}$ & energy state of the $i$-th DS at the beginning of time slot $t$ \\
\hline$\eta_{i, c}$ & charging efficiency coefficient of the $i$-th DS \\
\hline$\eta_{i, d}$ & discharging efficiency coefficient of the $i$-th DS \\
\hline$s_{i, \text { cap }}$ & energy capacity of the $i$-th DS \\
\hline$s_{i, \text { min }}$ & minimum preferred energy state of the $i$-th DS \\
\hline$s_{i, \text { max }}$ & maximum preferred energy state of the $i$-th DS \\
\hline$p_{m, t}$ & unit market electricity price at time slot $t$ \\
\hline$p_{m, \text { min }}$ & minimum unit market electricity price \\
\hline$p_{m, \text { max }}$ & maximum unit market electricity price \\
\hline$p_{c, t}$ & unite price for charging service at time slot $t$ \\
\hline$p_{d, t}$ & unite price for discharging service at time slot $t$ \\
\hline$D_{i, c}(\cdot)$ & degradation cost function of the $i$-th DS for charging \\
\hline$D_{i, d}(\cdot)$ & degradation cost function of the $i$-th DS for discharging \\
\hline$d_{i, l}$ & lower bound of the second derivatives of $D_{i, c}(\cdot)$ and $D_{i, d}(\cdot)$ \\
\hline$l_{i, u}$ & upper bound of the long-term degradation cost of the $i$-th DS \\
\hline$C_{s}(\cdot)$ & cost function for clearing energy surplus using external sources \\
\hline$C_{d}(\cdot)$ & cost function for clearing energy deficit using external sources \\
\hline$c_{l}$ & lower bound of the second derivatives of $C_{s}(\cdot)$ and $C_{d}(\cdot)$ \\
\hline
\end{tabular}

in Section VIII. Finally, we conclude in Section IX.

Notation: Denote $[x]_{a}^{b}$ as $\min \{\max \{x, a\}, b\}$, which projects a scalar $x$ onto the interval $[a, b]$; denote $\mathbb{R}$ as the set of real numbers; denote $\mathbf{1}(A)$ as the indicator function, which equals 1 (resp. 0 ) if the event $A$ is true (resp. false); for a function $F(\cdot)$, denote $F^{\prime}(\cdot)$ and $F^{\prime \prime}(\cdot)$ as its first derivative (or gradient) and second derivative, respectively; denote $F(\cdot)^{-1}$ as its inverse function. The main symbols in this paper are summarized in Table I.

\section{RELATED WORKS}

The real-time power balancing problem has been addressed in previous works using three different approaches: supply side management, demand side management, and controlled charging and discharging of DS units. Below, we survey these works and highlight our contribution.

\section{A. Supply Side and Demand Side Management}

Power balance may be achieved directly, on the supply side, by controlling dispatchable generators, or on the demand side, by scheduling flexible loads.

Supply side management: In [5] and [6], by assuming that all demand loads are critical and must be met, the authors provide real-time algorithms to optimally schedule the power output of dispatchable generators, so as to minimize the system cost. 
In particular, [5] focuses on the average system performance, while [6] emphasizes the worst-case system performance.

Demand side management: In [7], [16], and [17], realtime power balance is achieved by scheduling the demand loads of users, with the objective of minimizing the average system cost. Specifically, [7] proposes to optimally schedule the non-interruptible and deferrable loads of individual users within their deadlines. The problem is formulated as a Markov decision process (MDP) problem and is solved distributively. In addition, both [16] and [17] have developed their solutions under the framework of Lyapunov optimization, and considered joint scheduling of flexible load and storage usage. In [16], a centralized real-time algorithm is provided, while in [17], a gradient-based distributed real-time algorithm is suggested.

In our work, we consider the alternative of using an aggregator-DS system for real-time power balancing. The DS units are external to the supply-demand dichotomy and are used to clear the residual power imbalance after direct supply side and demand side management. Therefore, our work is complementary to those works mentioned above. Moreover, the DS units we consider are general as long as they are capable of charging and discharging.

The Lyapunov optimization technique used in the development of our real-time sub-problems shares some similarity with [16] and [17]. However, compared with [16] and [17], we focus on the unique characteristics of storage units and employ a more realistic model that takes into account the battery inefficiency and energy gain/loss under charging/discharging. Moreover, compared with the distributed implementation in [17], our distributed algorithm is enabled by a novel cost cushion parameter design, leading to provably faster convergence.

\section{B. Energy Storage Management in Aggregator-DS System}

There is a growing body of recent works on power balancing using DS units. Specific to the aggregator-DS system, which focuses on the interaction between the aggregator and DS units, most works adopt centralized control, with the objective of maximizing the profit of the aggregator or DS units [18] [22], or the social welfare of the system [23], [24]. To our best knowledge, the only previous works that address distributed control specific to the aggregator-DS system are presented in [25]-[27], all studying a static system. In particular, in [25], assuming that each DS unit considers only whether to charge, discharge, or remain idle, a service pricing function is developed so that the difference between the power imbalance amount and the sum DS contribution is minimized. The same goal is considered in [26], where each DS unit can additionally decide its charging and discharging amounts. A pricing strategy and a distributed consensus algorithm are designed for the DS units to reach a unique Nash equilibrium, but the optimality of the pricing strategy is not discussed. In [27], the objective is to minimize the system cost while aligning each DS unit interest with the system benefit. It considers both synchronous and asynchronous communication between the aggregator and each DS unit, but it adopts a greedy instantaneous allocation approach that ignores the opportunity of joint optimization over multiple time instances.

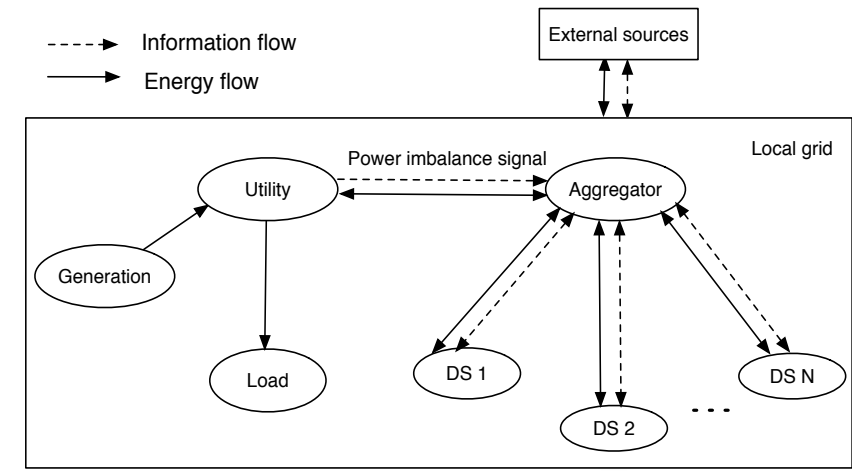

Fig. 1. Schematic representation of a local power grid.

In addition to the limitations summarized above, most of these earlier works have omitted to consider some essential characteristics of the aggregator-DS system. For example, a deterministic model is used in [18] and [21], which ignore the uncertainty of the electricity price, and the dynamics of the power imbalance amount is not incorporated in [25]-[27]. For the aggregator, the potential cost for using external energy sources to clear the imbalance is ignored in [18]-[22]. For DS units, the finite battery size constraints are not considered in [25]; the battery degradation costs due to frequent charging/discharging in real-time operation are omitted in [20][22], [25], [26]; the energy gain/loss in charging/discharging is ignored in [20], [23], [25], [26].

In this work, we consider all above factors missed in previous works in a more complete aggregator-DS system model. For the system to collectively help a power grid achieve power balance, we develop a real-time distributed algorithm that does not rely on any statistics of the system and is easy to implement in reality. Moreover, the proposed algorithm is proved to be asymptotically optimal as each DS unit capacity increases. We have previously studied realtime power balancing with an aggregator coordinating static and dynamic DS units in [23] and [24] respectively, with application to frequency regulation using electric vehicles. The main objective of those works is to fairly allocate the power imbalance amount among the participants. Furthermore, the aggregator centrally controls the charging and discharging of the electric vehicles. In this work, we consider a more realistic charging and discharging model with battery inefficiency and electricity prices, beyond others. More importantly, we aim to minimize the long-term system cost and propose a solution that can be solved collectively by the aggregator and all DS units in a distributed manner.

\section{System Model AND Problem StAtement}

We first describe an aggregator-DS system that provides real-time power balancing service to a power grid, and then formulate the problem.

\section{A. Power Balancing and Aggregator-DS System}

In a power grid as shown in Fig. 1, due to intrinsic prediction error of generation and load as well as the randomness of renewable sources, the generation amount cannot match the 
load amount continuously. The discrepancy between these two at any time can be represented by a power imbalance signal. Consider a time-slotted system with equal time intervals, which in practical systems may range from a few seconds to a few minutes. For ease of notation, we incorporate time into the power imbalance signal and use energy units below. At time slot $t$, we denote $g_{t},\left|g_{t}\right| \leq g_{\max }$, as the energy imbalance amount, which is random. If $g_{t}>0$, then the generation amount is greater than the load amount by $g_{t}$ units, which results in energy surplus. If $g_{t}<0$, then the generation amount is less than the load amount by $\left|g_{t}\right|$ units, which results in energy deficit. Define $\mathbf{1}_{s, t} \triangleq \mathbf{1}\left(g_{t}>0\right)$ and $\mathbf{1}_{d, t} \triangleq \mathbf{1}\left(g_{t}<0\right)$ as the indicators of energy surplus and energy deficit at time slot $t$, respectively, where $\mathbf{1}(\cdot)$ is the indicator function. Since energy surplus and energy deficit cannot happen simultaneously, we have $\mathbf{1}_{s, t} \cdot \mathbf{1}_{d, t}=0$.

Assume that an aggregator serves the power grid and employs energy storage, capable of charging and discharging, to clear the energy imbalance in every time slot. Since the magnitude of the energy imbalance signal, $\left|g_{t}\right|$, is in general large and building a massive energy storage unit could be costly, the aggregator instead coordinates $N$ (smaller) DS units, possibly owned by different users, to provide power balancing service. These DS units can be, for example, batteries in electric vehicles and batteries deployed at renewable generators. The number of such DS units is expected to be large in the near future owing to electrification of transportation and the integration of more and more renewable sources.

At the beginning of time slot $t$, the aggregator receives the energy imbalance signal $g_{t}$ from the utility. If $g_{t}>0$, the aggregator is required to absorb $g_{t}$ units of energy during time slot $t$. If $g_{t}<0$, the aggregator is required to contribute $\left|g_{t}\right|$ units of energy during time slot $t$. Upon receiving the energy imbalance signal, the aggregator communicates with each DS unit bidirectionally so as to negotiate the individual energy absorption or contribution amount. The information and energy flows of the system are depicted in Fig. 1. For simplicity of analysis, in the sequel we assume that all DS units are static and are connected to the aggregator all the time. Examples of such DS units are batteries deployed at renewable generators. The case that involves dynamic DS units, such as batteries in electric vehicles, will be discussed in Section VIII.

For the $i$-th DS unit, denote $x_{i, t} \geq 0$ as its charging amount during time slot $t$ in the case of energy surplus, and $y_{i, t} \geq 0$ as its discharging amount during time slot $t$ in the case of energy deficit. Because of limitation imposed by the charging/discharging circuit, $x_{i, t}$ and $y_{i, t}$ are upper bounded. For simplicity, assume that the maximum allowed charging and discharging amounts are of the same quantity, denoted by $r_{i, \max }$, i.e.,

$$
0 \leq x_{i, t} \leq r_{i, \max }, 0 \leq y_{i, t} \leq r_{i, \max } .
$$

Define $N$-dimensional charging and discharging amount vectors at time slot $t$ as $\mathbf{x}_{t} \triangleq\left[x_{1, t}, \cdots, x_{N, t}\right]$ and $\mathbf{y}_{t} \triangleq\left[y_{1, t}, \cdots, y_{N, t}\right]$, respectively.

Let $\eta_{i, c} \in(0,1]$ be the charging efficiency coefficient of the $i$-th DS unit, and $\eta_{i, d} \in[1, \infty)$ be the discharging efficiency coefficient. Because of the battery inefficiency, generally, the actual stored energy through charging is less than $x_{i, t}$, and the actual contributed energy through discharging is larger than $y_{i, t}$. Denote $s_{i, t}$ as the energy state of the $i$-th DS unit at the beginning of time slot $t$. Due to charging and discharging, the energy state $s_{i, t}$ fluctuates over time and evolves as follows ${ }^{1}$ :

$$
s_{i, t+1}=s_{i, t}+\mathbf{1}_{s, t} \eta_{i, c} x_{i, t}-\mathbf{1}_{d, t} \eta_{i, d} y_{i, t} \triangleq s_{i, t}+b_{i, t}
$$

where

$$
b_{i, t} \triangleq \mathbf{1}_{s, t} \eta_{i, c} x_{i, t}-\mathbf{1}_{d, t} \eta_{i, d} y_{i, t}
$$

is defined as the effective charging/discharging amount of the $i$-th DS unit during time slot $t$.

Charging a battery near its capacity or discharging it close to the zero energy state can significantly reduce battery lifetime [28]. Therefore, lower and upper bounds on the battery energy state are usually imposed by its manufacturer or owner. Denote $s_{i \text {,cap }}$ as the energy capacity of the $i$-th DS unit, and $\left[s_{i, \min }, s_{i, \max }\right]$ as its preferred energy range with $0 \leq s_{i, \min }<s_{i, \max } \leq s_{i \text {,cap }}$. We assume that the energy state at each time slot should be limited within the preferred range, i.e.,

$$
s_{i, \min } \leq s_{i, t} \leq s_{i, \max } .
$$

Combining the constraints (1) and (4), we can compactly represent the constraints of $x_{i, t}$ and $y_{i, t}$ as

$$
0 \leq x_{i, t} \leq \min \left\{r_{i, \max }, \frac{s_{i, \max }-s_{i, t}}{\eta_{i, \mathrm{c}}}\right\}
$$

and

$$
0 \leq y_{i, t} \leq \min \left\{r_{i, \max }, \frac{s_{i, t}-s_{i, \min }}{\eta_{i, \mathrm{~d}}}\right\},
$$

respectively.

Since a DS unit absorbs and contributes energy in charging and discharging, respectively, it has either energy gain or energy loss when providing real-time power balancing service. Denote the unit market electricity price at time slot $t$ as $p_{m, t} \in\left[p_{m, \min }, p_{m, \max }\right]$. Then, the revenue of the $i$-th DS unit for absorbing energy in the case of energy surplus is $p_{m, t} x_{i, t}$, and the loss for contributing energy in the case of energy deficit is $p_{m, t} \eta_{i, d} y_{i, t}$. Additionally, by providing power balancing service, each DS unit can receive payment from the aggregator for its controllable and flexible charging/discharging capability. Denote the unit prices for charging and discharging services at time slot $t$ as $p_{c, t}$ and $p_{d, t}$, respectively. Assume that the aggregator pays for the charging/discharging based on the actual service amounts $x_{i, t}$ and $y_{i, t}$. In other words, the $i$-th DS unit receives payment $p_{c, t} x_{i, t}$ in the case of energy surplus for charging, and payment $p_{d, t} y_{i, t}$ in the case of energy deficit for discharging. As a result, the effective cost of the $i$ th DS unit for providing power balancing service at time slot $t$ is

$\phi_{i, t} \triangleq \mathbf{1}_{s, t}\left(-p_{m, t} x_{i, t}-p_{c, t} x_{i, t}\right)+\mathbf{1}_{d, t}\left(p_{m, t} \eta_{i, d} y_{i, t}-p_{d, t} y_{i, t}\right)$.

For each DS unit, offering power balancing service is accompanied by battery degradation for frequent charging

\footnotetext{
${ }^{1}$ We assume that the role of the DS units is to exclusively provide real-time power balancing service when connected and thus do not explicitly consider their own charging needs.
} 
and extra cycling of battery [29]. Denote $D_{i, c}(\cdot)$ and $D_{i, d}(\cdot)$ as the degradation cost functions with respect to the charging amount and the discharging amount, respectively, with $D_{i, c}(0)=D_{i, d}(0)=0$. Since the actual discharging amount is $\eta_{i, d} y_{i, t}$, for notation simplicity, we will merge $\eta_{i, d}$ into the function $D_{i, d}(\cdot)$. Furthermore, since faster charging or discharging (larger value of $x_{i, t}$ or $y_{i, t}$ ) generally has a more detrimental effect on battery lifetime, $D_{i, c}(\cdot)$ and $D_{i, d}(\cdot)$ can be approximated by increasing convex functions in general. To facilitate later analysis, we slightly strengthen this condition and take the following assumptions:

C1:

- $D_{i, c}(\cdot)$ and $D_{i, d}(\cdot)$ are increasing, strictly convex, and twice continuously differentiable on $\left[0, r_{i, \max }\right]$.

- The second derivatives of $D_{i, c}(\cdot)$ and $D_{i, d}(\cdot)$ are lower bounded by a constant $d_{i, l}>0$ on $\left[0, r_{i, \max }\right]$.

To limit battery degradation, the $i$-th DS unit sets a predesigned upper bound $l_{i, u} \geq 0$ to restrict the longterm degradation cost, which can be formally expressed by $\lim _{T \rightarrow \infty} \frac{1}{T} \sum_{t=0}^{T-1} \mathbb{E}\left[\mathbf{1}_{s, t} D_{i, c}\left(x_{i, t}\right)+\mathbf{1}_{d, t} D_{i, d}\left(y_{i, t}\right)\right] \leq l_{i, u}$.

Due to a lack of participating DS units or high battery degradation cost, sometimes the sum contribution of all DS units may be insufficient to clear the total power imbalance amount. Specifically, for energy surplus, this insufficiency means that $\sum_{i=1}^{N} x_{i, t}<g_{t}$, and for energy deficit, it means that $\sum_{i=1}^{N} y_{i, t}<\left|g_{t}\right|$. Hence, from time to time, to fill the gap, the aggregator needs to exploit external energy sources, such as the external real-time electricity market ${ }^{2}$. Denote the cost functions of the external sources for clearing energy surplus and energy deficit as $C_{s}(\cdot)$ and $C_{d}(\cdot)$, respectively, with $C_{s}(0)=C_{d}(0)=0$. Then, the cost of the aggregator for exploiting the external sources at time slot $t$ can be represented as $\mathbf{1}_{s, t} C_{s}\left(g_{t}-\sum_{i=1}^{N} x_{i, t}\right)+\mathbf{1}_{d, t} C_{d}\left(\left|g_{t}\right|-\sum_{i=1}^{N} y_{i, t}\right)$. We assume the following conditions on the external cost functions:

C2:

- $C_{s}(\cdot)$ and $C_{d}(\cdot)$ are increasing, strictly convex, and twice continuously differentiable on $\left[0, g_{\max }\right]$.

- The second derivatives of $C_{s}(\cdot)$ and $C_{d}(\cdot)$ are lower bounded by a constant $c_{l}>0$ on $\left[0, g_{\max }\right]$.

Finally, the total cost of the aggregator, including that for using the external sources and the payment to all DS units, is given by

$$
\begin{aligned}
& \varphi_{t} \triangleq \mathbf{1}_{s, t} {\left[C_{s}\left(g_{t}-\sum_{i=1}^{N} x_{i, t}\right)+p_{c, t} \sum_{i=1}^{N} x_{i, t}\right] } \\
&+\mathbf{1}_{d, t}\left[C_{d}\left(\left|g_{t}\right|-\sum_{i=1}^{N} y_{i, t}\right)+p_{d, t} \sum_{i=1}^{N} y_{i, t}\right] .
\end{aligned}
$$

Combining the costs of all DS units with the cost of the aggregator, we have the total cost of the aggregator-DS system at time slot $t$ given by

$$
w_{t} \triangleq\left(\sum_{i=1}^{N} \phi_{i, t}\right)+\varphi_{t} .
$$

\footnotetext{
${ }^{2}$ In practice, the imbalance signal $g_{t}$ may relate to the capacity of the service provider. In this paper, we focus on the aggregator-DS system, and assume that $g_{t}$ is determined externally and the aggregator guarantees to clear the imbalance in every time slot.
}

Notice that the payment for the charging/discharging service does not appear in the expression of $w_{t}$. This is because such payment is transferred from the aggregator to the DS units, hence not affecting the system-wide cost. We will revisit the service payment in Section V-C.

\section{B. Problem Statement}

The aggregator is assumed to be regulated and non profitdriven. For example, it can represent a government-funded party that encourages the integration of DS units into a power grid. The aggregator coordinates the DS units to provide realtime power balancing service, and aims to minimize the longterm system cost while respecting the battery capacity and degradation cost constraints of each DS unit. We assume that each DS unit is willing to provide real-time power balancing service and is under contract with the aggregator. In return, the DS units will be paid for such a service as described in Section III-A ${ }^{3}$.

We formulate the real-time power balancing problem as the following stochastic optimization problem.

$$
\begin{array}{r}
\text { P1: } \min _{\left\{\mathbf{x}_{t}, \mathbf{y}_{t}\right\}} \quad \lim _{T \rightarrow \infty} \frac{1}{T} \sum_{t=0}^{T-1} \mathbb{E}\left[w_{t}\right] \\
\text { s.t. } \quad 0 \leq x_{i, t} \leq \min \left\{r_{i, \max }, \frac{s_{i, \max }-s_{i, t}}{\eta_{i, c}}\right\}, \forall i, t \\
0 \leq y_{i, t} \leq \min \left\{r_{i, \max }, \frac{s_{i, t}-s_{i, \min }}{\eta_{i, d}}\right\}, \forall i, t \\
\sum_{i=1}^{N} x_{i, t} \leq \mathbf{1}_{s, t} g_{t}, \quad \sum_{i=1}^{N} y_{i, t} \leq \mathbf{1}_{d, t}\left|g_{t}\right|, \forall t \\
\lim _{T \rightarrow \infty} \frac{1}{T} \sum_{t=0}^{T-1} \mathbb{E}\left[\mathbf{1}_{s, t} D_{i, c}\left(x_{i, t}\right)+\mathbf{1}_{d, t} D_{i, d}\left(y_{i, t}\right)\right] \leq l_{i, u}, \forall i
\end{array}
$$

where the expectations above are taken over the random system state defined as $A_{t} \triangleq\left(g_{t}, p_{m, t}\right)$ and the possibly random decisions $\left(\mathbf{x}_{t}, \mathbf{y}_{t}\right)$. The rationale for constraints (5)-(8) is given in Section III-A. By (7), we mean that, first, the sum contribution of all DS units should not exceed the required amount, and second, in the case of energy deficit (resp. energy surplus) the charging (resp. discharging) amount of each DS unit should be zero.

The above optimization problem can be solved centrally by traditional approaches such as dynamic programming [31], provided that the aggregator knows perfectly about the system statistics and can fully control the charging/discharging of all DS units. However, for one, dynamic programming is known to suffer from "the curse of dimensionality," and accurate statistics cannot be easily obtained in practice. For another, direct charging/discharging control not only overrides a DS owner's individual choice but also leads to high computational complexity as the number of participating DS units becomes large.

Motivated by these concerns, our goal in this paper is to develop a real-time distributed algorithm, by which the

\footnotetext{
${ }^{3}$ We emphasize that the market aspects, such as the contract design investigated in [30], are not the focus of this paper.
} 
statistics of the system state is not required and each DS unit is able to make its own decision. This is a challenging problem due to the presence of the dynamic system state, the finite battery size constraints, and the coupling of decisions among all DS units. To address this problem, we first decompose the long-term optimization problem P1 into real-time subproblems.

\section{Decomposition into ReAl-Time Sub-PRoblems}

To solve P1, we now propose the corresponding realtime sub-problems under the general framework of Lyapunov optimization [13], with modifications to handle finite battery size constraints and to facilitate the distributed algorithm introduced later.

\section{A. Problem Relaxation}

Recall that for each DS unit, the hard constraints of the charging/discharging amount, i.e., (5) and (6), are equivalent to the constraints (1) and (4). Due to the battery size constraint (4), for each DS unit, the current charging/discharging decision is coupled with all previous charging/discharging decisions through the current energy state, which complicates the optimization. To avoid such coupling, we replace (4) with a new time average constraint and introduce the following relaxed problem:

$$
\begin{array}{cl}
\text { P2: } & \min _{\left\{\mathbf{x}_{t}, \mathbf{y}_{t}\right\}} \\
\text { s.t. } & \lim _{T \rightarrow \infty} \frac{1}{T} \sum_{t=0}^{T-1} \mathbb{E}\left[w_{t}\right] \\
& (1)(7)(8), \\
& \lim _{T \rightarrow \infty} \frac{1}{T} \sum_{t=0}^{T-1} \mathbb{E}\left[b_{i, t}\right]=0, \forall i
\end{array}
$$

where $b_{i, t}$ is defined in (3). As opposed to (4), by which the energy state is always bounded, (9) requires that the effective charging/discharging amount is zero on average.

We now demonstrate that (4) implies (9), so that P2 is indeed a relaxation of $\mathbf{P 1}$. Summing both sides of the energy state equation (2) over $t \in\{0,1, \cdots, T-1\}$ and dividing them by $T$ yields

$$
\frac{s_{i, T}}{T}-\frac{s_{i, 0}}{T}=\frac{1}{T} \sum_{t=0}^{T-1} b_{i, t} .
$$

Taking expectations on both sides of (10) and taking limits over $T$ gives

$$
\lim _{T \rightarrow \infty} \frac{\mathbb{E}\left[s_{i, T}\right]}{T}-\lim _{T \rightarrow \infty} \frac{\mathbb{E}\left[s_{i, 0}\right]}{T}=\lim _{T \rightarrow \infty} \frac{1}{T} \sum_{t=0}^{T-1} \mathbb{E}\left[b_{i, t}\right] .
$$

Since $s_{i, T}$ and $s_{i, 0}$ are bounded by (4), the left hand side of (11) is equal to zero and the constraint (9) holds.

By removing the coupling in charging/discharging decisions due to the battery size constraints, the relaxed problem P2 allows us to apply Lyapunov optimization to decompose the original problem into real-time sub-problems. We will show later in Section VI that our developed solution in fact also satisfies (4), so it is feasible for P1. This relaxation technique to accommodate the type of time-coupled decision constraints such as (5) and (6) was first introduced in [32] for energy management in a data center equipped with an ideal battery, and later was also applied in [16] and [17]. Compared with [32], besides our problem being different from it, we consider multiple DS units. Compared with [16] and [17], the structure of our problem is more complicated, with a nonlinear objective which allows for bidirectional energy flow between the aggregator and DS units. Thus, it is more involved in the relaxation treatment to ensure that the battery size constraints are satisfied.

\section{B. Virtual Queue Design}

To solve P2, we introduce virtual queues and transform the time average constraints (8) and (9) to queue stability constraints, as explained below.

Consider constraint (8). To facilitate distributed implementation which will be explained later, we add a constant cost cushion $a_{i}>0$ to both sides of (8), and obtain the following equivalent constraint for each DS unit:

$$
\lim _{T \rightarrow \infty} \frac{1}{T} \sum_{t=0}^{T-1} \mathbb{E}\left[\mathbf{1}_{s, t} D_{i, c}\left(x_{i, t}\right)+\mathbf{1}_{d, t} D_{i, d}\left(y_{i, t}\right)+a_{i}\right] \leq \hat{l}_{i, u}
$$

where $\hat{l}_{i, u} \triangleq l_{i, u}+a_{i}$. Define a virtual queue $J_{i, t}$, which updates as

$$
\begin{aligned}
J_{i, t+1}=\max \left\{J_{i, t}-\hat{l}_{i, u}, 0\right\} & +\mathbf{1}_{s, t} D_{i, c}\left(x_{i, t}\right) \\
& +\mathbf{1}_{d, t} D_{i, d}\left(y_{i, t}\right)+a_{i} .
\end{aligned}
$$

Initialize $J_{i, 0}=a_{i}$ and define $\mathbf{J}_{t} \triangleq\left[J_{1, t}, \cdots, J_{N, t}\right]$. Based on (13), queue backlog $J_{i, t}$ accumulates the total amount of degradation cost in excess of $\hat{l}_{i, u}$. The function of $a_{i}$ is to guarantee that $J_{i, t} \geq a_{i}$. The introduction of $a_{i}$ is important, and we will discuss the design of $a_{i}$ in Section V-B.

For constraint (9), we associate it with a virtual queue $K_{i, t}$, which evolves as

$$
K_{i, t+1}=K_{i, t}+b_{i, t} .
$$

Define $\mathbf{K}_{t} \triangleq\left[K_{1, t}, \cdots, K_{N, t}\right]$. By (14), $K_{i, t}$ accumulates the total effective charging/discharging amount. Comparing (14) with (2), we can see that $K_{i, t}$ and the energy state $s_{i, t}$ evolve in the same manner. We relate them by initializing $K_{i, 0}=$ $s_{i, 0}-\beta_{i}$, where the shift parameter $\beta_{i}$ is set to be

$$
\beta_{i} \triangleq s_{i, \min }+\eta_{i, d} r_{i, \max }-V\left(p_{m, \min }-\frac{c_{\max }}{\eta_{i, d}}\right),
$$

where $c_{\max } \triangleq \max \left\{C_{s}^{\prime}\left(g_{\max }\right), C_{d}^{\prime}\left(g_{\max }\right)\right\}$ and the weight $V \in$ $\left(0, V_{\max }\right]$ with

$$
V_{\max } \triangleq \min _{1 \leq i \leq N}\left\{\frac{s_{i, \max }-s_{i, \min }-\left(\eta_{i, c}+\eta_{i, d}\right) r_{i, \max }}{\frac{c_{\max }+p_{m, \max }}{\eta_{i, c}}+\frac{c_{\max }}{\eta_{i, d}}-p_{m, \min }}\right\} .
$$

Thus, the virtual queue $K_{i, t}$ is a shifted version of the energy state $s_{i, t} . K_{i, t}$ is introduced to track $s_{i, t}$. More importantly, as we will see later, the boundedness of $s_{i, t}$ can be guaranteed 
through the control of $K_{i, t}$. The design of $\beta_{i}$ and $V_{\max }$ in (15) and (16) is crucial. We will show in Section VI-B how the constraint (4) can be guaranteed by such design.

Note that under the real-time operation, the value of $r_{i, \max }$ in (16) is generally much smaller than the energy capacity. For example, for the 2012 Ford Focus Electric, the energy capacity is $23 \mathrm{kWh}$ and the maximum charging/discharging rate is $6.6 \mathrm{~kW}$. Assuming that the duration of each time slot is 5 minutes, we then have $r_{i, \max }=0.55 \mathrm{kWh} \ll 23 \mathrm{kWh}$. By this observation, from (16), we have $V_{\max }>0$ in general.

Finally, we show that the time-averaged constraints (8) and (9) can be transformed into the mean rate stability constraints of virtual queues, which is a direct result from [13].

Lemma 1: Constraints (8) and (9) hold if the virtual queues $J_{i, t}$ and $K_{i, t}$ are mean rate stable, respectively.

\section{Real-Time Sub-Problems}

At time slot $t$, define a vector $\boldsymbol{\Theta}_{t} \triangleq\left[\mathbf{J}_{t}, \mathbf{K}_{t}\right]$, the Lyapunov function $L\left(\boldsymbol{\Theta}_{t}\right) \triangleq \frac{1}{2} \sum_{i=1}^{N}\left(J_{i, t}^{2}+K_{i, t}^{2}\right)$, and the associated oneslot Lyapunov drift

$$
\Delta\left(\boldsymbol{\Theta}_{t}\right) \triangleq \mathbb{E}\left[L\left(\boldsymbol{\Theta}_{t+1}\right)-L\left(\boldsymbol{\Theta}_{t}\right) \mid \boldsymbol{\Theta}_{t}\right] .
$$

The drift-plus-cost function is defined as $\Delta\left(\boldsymbol{\Theta}_{t}\right)+V \mathbb{E}\left[w_{t} \mid \boldsymbol{\Theta}_{t}\right]$ [13], in which the time-averaged constraints and the objective function are jointly considered, with the weight $V$ (the same $V$ as in (15)) controlling their trade-off. In the following proposition, we provide an upper bound on the drift-plus-cost function.

Proposition 1: For all possible policies of the charging/discharging decisions of all DS units, and all possible values of $\boldsymbol{\Theta}_{t}$, the drift-plus-cost function is upper bounded as follows:

$$
\begin{aligned}
& \Delta\left(\boldsymbol{\Theta}_{t}\right)+V \mathbb{E}\left[w_{t} \mid \boldsymbol{\Theta}_{t}\right] \leq B+V \mathbb{E}\left[w_{t} \mid \boldsymbol{\Theta}_{t}\right]+\sum_{i=1}^{N} K_{i, t} \mathbb{E}\left[b_{i, t} \mid \boldsymbol{\Theta}_{t}\right] \\
& +\sum_{i=1}^{N} J_{i, t} \mathbb{E}\left[\mathbf{1}_{s, t} D_{i, c}\left(x_{i, t}\right)+\mathbf{1}_{d, t} D_{i, d}\left(y_{i, t}\right)-l_{i, u} \mid \boldsymbol{\Theta}_{t}\right]
\end{aligned}
$$

where

$$
\begin{aligned}
B \triangleq \frac{1}{2} \sum_{i=1}^{N}\left[\hat{l}_{i, u}^{2}\right. & +\left(\max \left\{D_{i, c}\left(r_{i, \max }\right), D_{i, d}\left(r_{i, \max }\right)\right\}+a_{i}\right)^{2} \\
& \left.+r_{i, \max }^{2}\right],
\end{aligned}
$$

and $V \in\left(0, V_{\max }\right]$.

Proof: See Appendix A.

Adopting the general framework of Lyapunov optimization [13], we design a real-time algorithm to minimize the upper bound of the drift-plus-cost function on the right hand side of (17). The algorithm can lead to a guaranteed performance as shown in Section VI. Consequently, we consider the real-time sub-problems for energy surplus and energy deficit at each time slot $t$ as follows. For notation simplicity, we will omit the subscript $t$ of the optimization variables whenever it is clear from the context.
P2(a) (energy surplus):

$$
\begin{aligned}
& \min _{\mathbf{x}}\left[\sum_{i=1}^{N} J_{i, t} D_{i, c}\left(x_{i}\right)-V p_{m, t} x_{i}+K_{i, t} \eta_{i, c} x_{i}\right] \\
& \quad+V C_{s}\left(g_{t}-\sum_{i=1}^{N} x_{i}\right) \\
& \text { s.t. } \quad 0 \leq x_{i} \leq r_{i, \max }, \quad \sum_{i=1}^{N} x_{i} \leq g_{t} .
\end{aligned}
$$

P2(b) (energy deficit):

$$
\begin{aligned}
& \min _{\mathbf{y}}[\left.\sum_{i=1}^{N} J_{i, t} D_{i, d}\left(y_{i}\right)+V p_{m, t} \eta_{i, d} y_{i}-K_{i, t} \eta_{i, d} y_{i}\right] \\
&+V C_{d}\left(\left|g_{t}\right|-\sum_{i=1}^{N} y_{i}\right) \\
& \text { s.t. } \quad 0 \leq y_{i} \leq r_{i, \max }, \quad \sum_{i=1}^{N} y_{i} \leq\left|g_{t}\right| .
\end{aligned}
$$

The optimization variables $\mathbf{x}$ and $\mathbf{y}$ are $N$-dimensional vectors with the $i$-th element being $x_{i}$ and $y_{i}$, respectively.

\section{Distributed Algorithm for Real-Time SUB-PROBLEMS}

The real-time sub-problems P2(a) and P2(b) can be solved by the aggregator in a centralized way. However, since the DS units may belong to different users, they may not be willing to relinquish direct control of charging/discharging to the aggregator. In addition, the computational complexity of centralized control would grow too quickly as the number of DS units increases. In this section, we employ Lagrange dual decomposition and adapt a fast iterative algorithm to solve P2(a) and P2(b) distributively. Since energy surplus and energy deficit cannot happen simultaneously and their analyses are similar, in the following, we focus on the energy surplus problem P2(a).

\section{A. Lagrange Dual Decomposition}

In P2(a), since $J_{i, t} \geq a_{i}>0$ and $D_{i, c}(\cdot)$ is strictly convex, the objective function is strictly convex, which means that there is at most one global minimizer. Additionally, since the objective function is continuous and the constraint set of $\mathbf{x}$ is compact, there is at least one minimizer. Therefore, $\mathbf{P 2 ( a ) ~ h a s ~}$ a unique solution.

However, we note that the term $C_{s}\left(g_{t}-\sum_{i=1}^{N} x_{i}\right)$ in the objective function and the term $\sum_{i=1}^{N} x_{i} \leq g_{t}$ in the constraint are functions of the charging amounts of all DS units, which hinders a distributed algorithm. To avoid such coupling, we first introduce an auxiliary variable $q \in\left[0, g_{t}\right]$ to represent the difference between the energy imbalance amount and the sum contribution of all DS units, i.e., $g_{t}-\sum_{i=1}^{N} x_{i}$, and consider the following problem.

\section{P2(a'):}

$\min _{\mathbf{x}, q}\left[\sum_{i=1}^{N} J_{i, t} D_{i, c}\left(x_{i}\right)-V p_{m, t} x_{i}+K_{i, t} \eta_{i, c} x_{i}\right]+V C_{s}(q)$ 


$$
\begin{array}{ll}
\text { s.t. } & 0 \leq x_{i} \leq r_{i, \max }, \quad 0 \leq q \leq g_{t}, \\
& \sum_{i=1}^{N} x_{i}+q=g_{t} .
\end{array}
$$

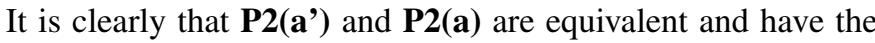
same unique solution in $\mathbf{x}$.

Next we associate the equality constraint (20) with a Lagrange multiplier $\lambda$. The partial Lagrangian of P2(a') is

$$
\begin{aligned}
F_{t}(\mathbf{x}, q, \lambda)= & {\left[\sum_{i=1}^{N} J_{i, t} D_{i, c}\left(x_{i}\right)-V p_{m, t} x_{i}+K_{i, t} \eta_{i, c} x_{i}\right] } \\
& +V C_{s}(q)+\lambda\left(g_{t}-\sum_{i=1}^{N} x_{i}-q\right) .
\end{aligned}
$$

The dual function $G_{t}(\lambda)$ is defined as the partial minimum of $F_{t}(\mathbf{x}, q, \lambda)$ with respect to the primal variables $\mathbf{x}$ and $q$ :

$$
G_{t}(\lambda)=\min _{\mathbf{x}, q} \quad F_{t}(\mathbf{x}, q, \lambda) \quad \text { s.t. } \quad(19) .
$$

Note that $G_{t}(\lambda)$ can be naturally decomposed into subproblems for each DS unit and the aggregator. Specifically, with $G_{t}(\lambda)$ divided by $V$, the sub-problem for each DS unit is

$$
\begin{array}{ll}
\min _{x_{i}} & -p_{m, t} x_{i}-\frac{\lambda}{V} x_{i}+\frac{J_{i, t}}{V} D_{i, c}\left(x_{i}\right)+\frac{K_{i, t} \eta_{i, c}}{V} x_{i} \\
\text { s.t. } & 0 \leq x_{i} \leq r_{i, \max }
\end{array}
$$

while the sub-problem for the aggregator is

$$
\min _{q} C_{s}(q)+\frac{\lambda}{V}\left(g_{t}-q\right) \quad \text { s.t. } \quad 0 \leq q \leq g_{t} .
$$

In (21), by interpreting $\frac{\lambda}{V}$ as $p_{c, t}$, the unit price for charging service as defined in Section III-A, we can view the objective of the $i$-th DS unit as minimizing the weighted sum of its different costs. By the Karush-Kuhn-Tucker (KKT) conditions, given $\lambda$, we obtain the unique solution of (21) in closed form: $\left[\left(D_{i, c}^{\prime}\right)^{-1}\left(\frac{V p_{m, t}+\lambda-K_{i, t} \eta_{i, c}}{J_{i, t}}\right)\right]_{0}^{r_{i, \max }}$. In the optimization problem (22), the aggregator minimizes its cost, including the external energy cost and the payment to all DS units. Again, the unique solution of (22) is found in closed form: $\left[\left(C_{s}^{\prime}\right)^{-1}\left(\frac{\lambda}{V}\right)\right]_{0}^{g_{t}}$. Thus, for any given $\lambda$, there is a unique solution for both (21) and (22). Consequently, the dual function $G_{t}(\lambda)$ is continuously differentiable in $\mathbb{R}[33]$.

The Lagrange dual problem is defined as the maximization of the dual function:

$$
\max _{\lambda} G_{t}(\lambda)
$$

Denote the optimal solution of the dual problem at time slot $t$ as $\lambda_{t}^{*}$, and the unique optimal solution of P2(a') at time slot $t$ as $\left(\mathbf{x}_{t}^{*}, q_{t}^{*}\right)$. Verifying Slater's condition on P2(a'), we are assured to have strong duality between the primal P2(a') and its dual (23) [14]. Thus, at time slot $t$, using $\lambda_{t}^{*}$, we can recover the optimal solution $\left(\mathbf{x}_{t}^{*}, q_{t}^{*}\right)$ by solving the sub-problems (21) and (22) [33].

To solve (23), we propose a fast iterative algorithm presented in the next subsection.

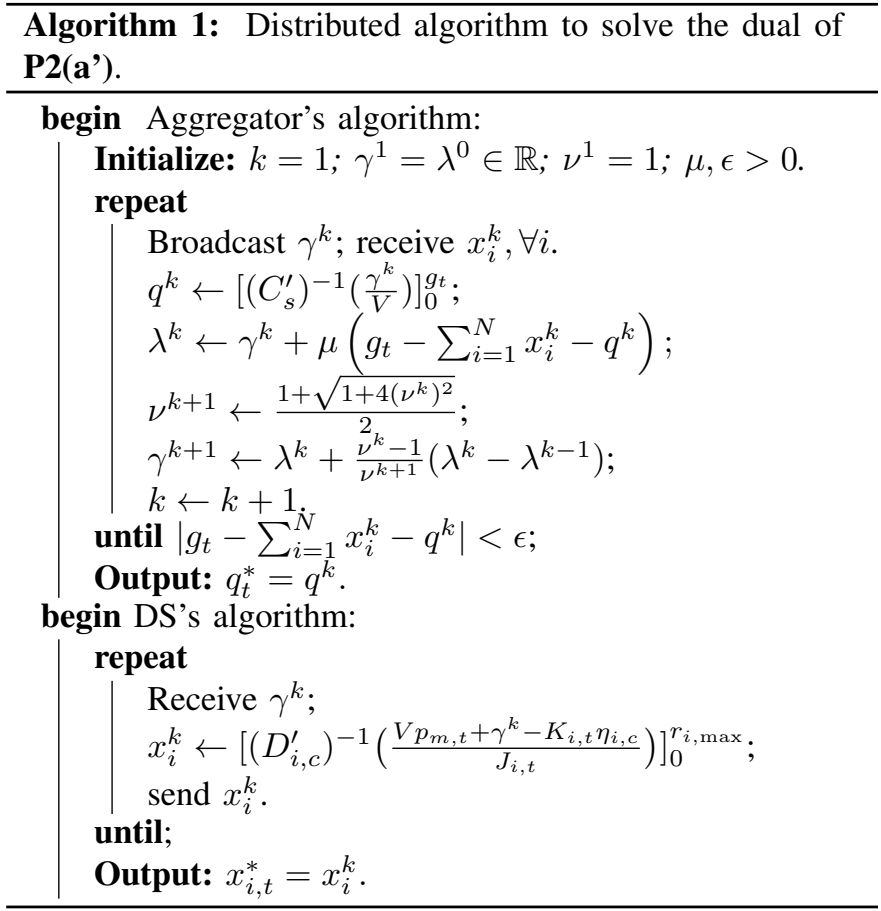

\section{B. Dual Maximization with FISTA and Convergence Analysis}

Since we consider the real-time power balancing problem with a short time interval, it is highly desirable that the algorithm can converge quickly in each time slot. To this end, we adapt a fast iterative shrinkage-thresholding algorithm (FISTA) [15] to solve the dual problem (23). The proposed algorithm is summarized in Algorithm 1. Compared with the standard gradient algorithm in which the Lagrange multiplier $\lambda^{k}$ is updated from the previous iterate $\lambda^{k-1}$, in Algorithm $1, \lambda^{k}$ is updated from $\gamma^{k}$, which is designed as a linear combination of the previous two iterates $\lambda^{k-1}$ and $\lambda^{k-2}$. Nonetheless, the extra computation is marginal.

Below we show that the gradient of the dual function is Lipschitz continuous, and determine its Lipschitz constant. The result is crucial for the convergence analysis of Algorithm 1.

Lemma 2: Under the conditions $\mathbf{C 1}$ and $\mathbf{C 2}$, the gradient of the dual function is Lipschitz continuous, i.e., we have $\left|G_{t}^{\prime}\left(\lambda_{1}\right)-G_{t}^{\prime}\left(\lambda_{2}\right)\right| \leq \rho\left|\lambda_{1}-\lambda_{2}\right|$ for all $\lambda_{1}, \lambda_{2} \in \mathbb{R}$, where the Lipschitz constant $\rho$ is given by

$$
\rho \triangleq(N+1) \max \left\{\frac{1}{a_{1} d_{1, l}}, \cdots, \frac{1}{a_{N} d_{N, l}}, \frac{1}{V c_{l}}\right\},
$$

where $d_{i, l}$ and $c_{l}$ are given in $\mathbf{C 1}$ and $\mathbf{C 2}$, respectively, and $a_{i}$ is the cost cushion parameter in (12).

Proof: See Appendix B.

In (24), since $a_{i}>0$, we have $0<\rho<\infty$. Using Lemma 2, we now prove the convergence of Algorithm 1.

Theorem 1: Under the conditions $\mathbf{C 1}$ and $\mathbf{C 2}$, in Algorithm 1 , with step size $\mu \in\left(0, \mu_{0}\right]$ where $\mu_{0} \triangleq 1 / \rho$, the sequence $\left\{\lambda^{k}\right\}$ converges to the optimum $\lambda_{t}^{*}$ of the dual problem (23). Furthermore, for any $k \geq 1$,

$$
G_{t}\left(\lambda_{t}^{*}\right)-G_{t}\left(\lambda^{k}\right) \leq \frac{2\left|\lambda^{0}-\lambda_{t}^{*}\right|^{2}}{\mu(k+1)^{2}},
$$


where $\lambda^{0}$ is the initial value of $\lambda$.

Proof: Given Lemma 2, the proof is similar to that in [15] with minor modification. See Appendix C.

Theorem 1 suggests that Algorithm 1 has a worst-case convergence rate of $O\left(1 / k^{2}\right)$. In comparison, the standard gradient algorithm, which is used in $[17]^{4}$ among many others, has a worst-case convergence rate $O(1 / k)$. Also from Theorem 1 , the step size $\mu$ is upper bounded by $\mu_{0}$, the inverse of the Lipschitz constant $\rho$. Based on the definition of $\rho$, we roughly have that, the larger the number of DS units, the smaller $\mu_{0}$ hence the slower the algorithm, which conforms to our intuition.

Furthermore, from (24), $\mu_{0}$ is a strictly increasing function of the cost cushion $a_{i}$ in the interval $\left(0, \frac{V c_{l}}{d_{i, l}}\right]$. Therefore, for the sole purpose of faster convergence, a larger $a_{i}$ should be chosen. However, later in Section VI-C, we will show that using a smaller $a_{i}$ may decrease the system cost.

\section{Price Signaling $p_{c, t}$}

We now look at the property of the optimal charging price signal $p_{c, t}^{*}=\frac{\lambda_{t}^{*}}{V}$. Since DS units have energy gain by charging, $\frac{\lambda_{t}^{*}}{V_{*}}$ can be negative. Below, we give a condition under which $\frac{\lambda_{t}^{*}}{V}$ is lower bounded and there exists a DS unit willing to provide power balancing service.

Proposition 2: At time slot $t$, if there exists a DS unit $j$ such that $-p_{m, t}+\frac{J_{j, t}}{V} D_{j, c}^{\prime}(x)+\frac{K_{j, t} \eta_{j, c}}{V}<C_{s}^{\prime}(x), \forall x \in(0, \epsilon)$, where $\epsilon$ is an arbitrarily small positive number, then the price signal $\frac{\lambda_{t}^{*}}{V}$ is lower bounded as

$$
\frac{\lambda_{t}^{*}}{V}>\min _{1 \leq i \leq N}\left\{-p_{m, t}+\frac{J_{i, t}}{V} D_{i, c}^{\prime}(0)+\frac{K_{i, t} \eta_{i, c}}{V}\right\}
$$

and the charging amount $x_{j, t}^{*}>0$.

Proof: See Appendix D.

Proposition 2 essentially states that, as long as there is a DS unit whose effective marginal cost, considering both the energy gain and the charging/discharging cost, is strictly less than the marginal cost of the external energy source, it is beneficial for the aggregator to incentivize the DS units to provide power balancing service (even though the price signal can be negative).

\section{Main Algorithm And Performance Optimality}

In this section, we give the main algorithm that combines the components presented in Sections IV and V, and analyze its performance with respect to our original problem $\mathbf{P 1}$.

\section{A. Main Algorithm}

In Algorithm 2, we formally state the real-time distributed algorithm for the aggregator-DS system to provide power balancing service. For presentation simplicity, we focus on the energy surplus case only.

We now discuss the information required in Algorithm 2 and show that Algorithm 2 can be easily implemented in

\footnotetext{
${ }^{4}$ The subgradient algorithm used in [17] reduces to the gradient algorithm when the dual function is differentiable.
}

Algorithm 2: Main algorithm: real-time distributed algo-

rithm for power balancing.

begin Aggregator's algorithm:

Repeat at each time:

1) Observe $g_{t}$.

2) Broadcast $\mathbf{1}_{s, t}$.

3) Execute aggregator's algorithm in Algorithm 1.

begin DS's algorithm:

Initialize: $J_{i, 0}=a_{i} ; K_{i, 0}=s_{i, 0}-\beta_{i}$.

Repeat at each time:

1) Observe $p_{m, t}, \mathbf{1}_{s, t}, J_{i, t}$, and $K_{i, t}$.

2) Execute DS's algorithm in Algorithm 1.

3) Update $J_{i, t}$ and $K_{i, t}$ based on (13) and (14), respectively.

practice. In Algorithm 2, the statistical information of the system is not required, and only instantaneous observations are needed, which can be obtained either locally or through simple communication. Specifically, at each time slot $t$, the aggregator observes the energy imbalance signal $g_{t}$, and each DS unit observes the electricity price $p_{m, t}$, the indicator of the energy imbalance signal $\mathbf{1}_{s, t}$, and the queue backlogs $J_{i, t}$ and $K_{i, t}$. To initialize $J_{i, 0}$ and $K_{i, 0}$ at each DS unit, the aggregator broadcasts $V, c_{l}$, and $c_{\max }$ to all DS units at the initial time. For the aggregator to determine $V_{\max }$ in (16), the values of $\eta_{i, c}, \eta_{i, d}$, and $s_{i, \max }-s_{i, \min }-\left(\eta_{i, c}+\eta_{i, d}\right) r_{i, \max }$ at each DS unit are required. In practice, however, it may be unnecessary to acquire all such information for determining $V_{\max }$. Note that as argued in Section IV-B the maximum allowed charging/discharging amount $r_{i, \max }$ is much smaller compared with the energy capacity. Thus, when the battery has high charging/discharging efficiency, i.e., $\eta_{i, c}$ and $\eta_{i, d}$ are close to 1 , approximately only the minimum battery capacity among all DS units is required for the design of $V_{\max }$.

\section{B. Boundedness of Energy State}

The proposed algorithm in Algorithm 2 is designed for P2, in which the battery size constraint (4) in P1 is replaced by the relaxed time average constraint (9). Thus, with $\left\{\mathbf{x}_{t}^{*}, \mathbf{y}_{t}^{*}\right\}$, it is not yet certain whether the resultant $s_{i, t}$ violates the battery size constraint (4), thus becoming infeasible for P1. We now demonstrate that under the proposed algorithm, $s_{i, t}$ in fact always satisfies (4).

Since the virtual queue $K_{i, t}$ is designed to be a shifted version of $s_{i, t}$, to prove the boundedness of $s_{i, t}$, it suffices to show that $K_{i, t}$ is restricted within a shifted preferred range. We first show through the following lemma that, $K_{i, t}$ is bounded for any initial value $K_{i, 0}$.

Lemma 3: For any initial value $K_{i, 0}$,

1) if $g_{t}>0$ and $K_{i, t}>V \frac{\left(p_{m, \max }+c_{\max }\right)}{\eta_{i, c}}, x_{i, t}^{*}=0$;

2) if $g_{t}<0$ and $K_{i, t}<V\left(p_{m, \min }-\frac{c_{\max }}{\eta_{i, d}}\right), y_{i, t}^{*}=0$.

Proof: See Appendix E.

Lemma 3 says that, given any $K_{i, 0}$, for energy surplus, if $K_{i, t}$ is greater than the above threshold, the resultant charging amount is zero, and thus $K_{i, t+1}$ cannot be increased at the next time slot. Similarly, for energy deficit, if $K_{i, t}$ is less than 
the above threshold, the resultant discharging amount is zero, and thus $K_{i, t+1}$ cannot be decreased at the next time slot. Therefore, $K_{i, t}$ is bounded.

Using Lemma 3, we next show that by our designed initialization, $K_{i, t}$ is bounded within a shifted preferred range.

Lemma 4: Given $K_{i, 0}=s_{i, 0}-\beta_{i}$, where $\beta_{i}$ is defined in (15), the queue backlog $K_{i, t}$ is bounded within $\left[s_{i, \min }-\right.$ $\left.\beta_{i}, s_{i, \max }-\beta_{i}\right]$ for all time slot $t$.

Proof: See Appendix F.

Remarks on Choices of $\beta_{i}$ and $V_{\max }$ : To track the energy state $s_{i, t}$, in principle, the shift $\beta_{i}$ could be any value. However, as required in Case $2^{\prime}$ of the proof of Lemma 4, $\beta_{i}$ should be lower bounded, i.e., $\beta_{i}=s_{i, \min }+\eta_{i, d} r_{i, \max }-$ $V\left(p_{m, \min }-\frac{c_{\max }}{\eta_{i, d}}\right)+\epsilon_{1}$ for any $\epsilon_{1} \geq 0$. Furthermore, as required in Case 1 of the proof, it is sufficient to set $V_{\max }=$ $\min _{1 \leq i \leq N}\left\{\frac{s_{i, \max }-s_{i, \min }-\eta_{i, d} r_{i, \max }-\epsilon_{1}-\epsilon_{2}}{\frac{c_{\max }+p_{m, \max }}{\eta_{i, c}}+\frac{c_{\max }}{\eta_{i, d}}-p_{m, \min }}\right\}$ with any $\epsilon_{2}>0$. Finally, to facilitate Case 2 of the proof, we set $\epsilon_{1}$ and $\epsilon_{2}$ to be 0 and $\eta_{i, c} r_{i, \max }$, respectively, to make $V_{\max }$ as large as possible. (As shown in Theorems 2 and 3 below, a larger $V_{\max }$ implies better performance by the proposed algorithm.) This leads to the specific designs as shown in (15) and (16).

By Lemma 4, the boundedness of the energy state $s_{i, t}$ is straightforward, and is given in the following lemma.

Lemma 5: Under the proposed algorithm, the energy state $s_{i, t}$ is bounded within $\left[s_{i, \min }, s_{i, \max }\right]$ for all time slot $t$.

\section{Optimality Analysis}

Denote the long-term system cost under the proposed algorithm as $f^{*}$ and that under the optimal solution for $\mathbf{P 1}$ as $f^{\text {opt }}$. Note that the optimal solution may require statistical information of the system. The optimality of the proposed algorithm is described in Theorems 2 and 3.

Theorem 2: Suppose that the system state $A_{t}$ is i.i.d. over time.

1) The virtual queues $J_{i, t}$ and $K_{i, t}$ are mean rate stable, and $\left\{\mathbf{x}_{t}^{*}, \mathbf{y}_{t}^{*}\right\}$ is feasible for $\mathbf{P 1}$;

2) $f^{*} \leq f^{\text {opt }}+\frac{B}{V}$, where $B$ is defined in (18) and $V \in$ $\left(0, V_{\max }\right]$.

Proof: See Appendix G.

From Theorem 2, the system cost under the proposed algorithm is away from the optimum by $O(1 / V)$. Thus, the larger $V$, the better the performance of the proposed algorithm. However, in practice, due to the boundedness of the preferred energy range, $V$ cannot be arbitrarily large and is upper bounded by $V_{\max }$, whose design rationale is given in the remarks after Lemma 4.

Based on the definition of $V_{\max }$ in (16), $V_{\max }$ increases with the smallest span of the DS units' preferred ranges, i.e., $\min _{1 \leq i \leq N}\left\{s_{i, \max }-s_{i, \min }\right\}$. Therefore, roughly speaking, the performance gap between the proposed algorithm and the optimum decreases as the smallest battery capacity increases. Asymptotically, as each DS unit's battery capacity goes to infinity, the proposed algorithm achieves the optimum.

We also note that the cost cushion $a_{i}$ increases the performance bound through the constant $B$. Hence, a smaller $a_{i}$ is desirable. Combining this with the convergence analysis in
Section V-B, we observe the important role of $a_{i}$ as a tuning parameter in the trade-off between system performance and convergence speed.

In Theorem 2, the i.i.d. condition of $A_{t}$ can be relaxed to Markovian, and a similar performance bound can be obtained. This allows us to design aggregator-DS systems in the case when the power imbalance amount $g_{t}$ and the electricity price $p_{m, t}$ are Markovian over time.

Theorem 3: Suppose that the system state $A_{t}$ evolves based on a finite state irreducible and aperiodic Markov chain.

1) The virtual queues $J_{i, t}$ and $K_{i, t}$ are mean rate stable, and $\left\{\mathbf{x}_{t}^{*}, \mathbf{y}_{t}^{*}\right\}$ is feasible for $\mathbf{P 1}$;

2) $f^{*} \leq f^{\text {opt }}+O(1 / V)$, where $V \in\left(0, V_{\max }\right]$.

Proof: The above results can be proved by expanding the proof of Theorem 2 using a multi-slot drift technique [13]. We omit the proof here for brevity.

\section{NUMERICAL ANALYSIS}

In the previous section, we have proven the analytical performance bound and asymptotic optimality of the proposed algorithm. In this section, we present numerical evaluation of the algorithm. Since as explained in Section II, the previous works on power balancing with the distributed aggregator-DS system, e.g., [25]-[27], study system models that are different and simpler than ours, they are not applicable for numerical comparison. Instead, we consider a greedy algorithm as a benchmark.

\section{A. Simulation Setup}

We have developed an aggregator-DS model in Matlab. Unless otherwise specified, the following parameters are set as default. The aggregator is connected with $N=150$ DS units, each with energy capacity $s_{i \text {,cap }}=23 \mathrm{kWh}$ and maximum charging/discharging rate $6.6 \mathrm{~kW}$ (based on the 2012 Ford Focus Electric). The duration of each time slot is $\Delta t=30$ seconds. Then, the maximum allowed charging/discharging amount of each DS unit is $r_{i, \max }=\frac{6.6 \Delta t}{3600} \mathrm{kWh}$. The charging and discharging efficiency coefficients are $\eta_{i, c}=0.8$ and $\eta_{i, d}=1.2$, respectively. The preferred energy range of each DS unit is $\left[0.1 s_{i, \text { cap }}, 0.9 s_{i, \text { cap }}\right]$, from which the initial energy state $s_{i, 0}$ is uniformly drawn. The degradation cost functions of the charging/discharging amount are $D_{i, c}(x)=$ $D_{i, d}(x)=x^{1.5}$, and the upper bound $l_{i, u}=\left(\frac{r_{i, \max }}{2}\right)^{1.5}$. The energy imbalance signal $g_{t}$ is i.i.d. over time and is sampled uniformly from $\left[-g_{\max }, g_{\max }\right]$, where $g_{\max }=\sum_{i=1}^{N} r_{i, \max }$. The unit market electricity price is 7 cents $/ \mathrm{kWh}$, which is the current off-peak electricity price in Ontario [34]. The external cost functions for clearing energy surplus and energy deficit are $C_{s}(x)=C_{d}(x)=7 x^{1.2}$. To determine the charging/discharging amounts of DS units at each time slot $t$, we apply the proposed algorithm in Algorithm 2 with $V=V_{\max }$. The cost cushion parameter $a_{i}=\frac{V_{\max } c_{l}}{d_{i, l}}$ by default for fast convergence. 
TABLE II

NUMBER OF ITERATIONS FOR $\left|g_{t}-\sum_{i=1}^{N} x_{i}^{k}-q^{k}\right|<0.01$

\begin{tabular}{l|l|l|l|l|l}
\hline$\mu / \mu_{0}$ & 1 & 10 & 20 & 50 & 100 \\
\hline$a_{i}=V_{\max } c_{l} / d_{i, l}$ & 279 & 105 & 85 & 45 & 26 \\
\hline$a_{i}=V_{\max } c_{l} /\left(4 d_{i, l}\right)$ & 964 & 411 & 183 & 131 & 44 \\
\hline
\end{tabular}

\section{B. Convergence of Distributed Algorithm}

In Table II, we show the convergence speed of Algorithm 1 by listing the number of iterations required for $\left|g_{t}-\sum_{i=1}^{N} x_{i}^{k}-q^{k}\right|<0.01$, when the current energy imbalance signal $g_{t}=g_{\max }$. From Table II, when the step size $\mu=\mu_{0}$, with the default $a_{i}$, the algorithm converges within 279 steps; in contrast, when $a_{i}$ is a quarter of the default value, the algorithm takes 964 steps to converge as the applied $\mu_{0}$ now is much smaller. We also observe that the convergence speed can be significantly improved by increasing $\mu$, indicating the robustness of the algorithm to the step size design.

\section{Comparison with Greedy Algorithm}

As a benchmark, we consider a greedy algorithm that is applied to the same system model as ours but aims to independently minimize the system cost at each time slot. Specifically, the charging/discharging amounts of the DS units under the greedy algorithm is determined by the following optimization problem at each time slot $t$.

$$
\begin{array}{cl}
\min _{\mathbf{x}_{t}, \mathbf{y}_{t}} & w_{t} \\
\text { s.t. } & (5)(6)(7), \mathbf{1}_{s, t} D_{i, c}\left(x_{i, t}\right)+\mathbf{1}_{d, t} D_{i, d}\left(y_{i, t}\right) \leq l_{i, u}, \forall i .
\end{array}
$$

The above problem produces a feasible solution for $\mathbf{P 1}$ at each time $t$ and can be implemented distributively following the technique in Section V.

In Figs. 2 and 3, we compare the proposed algorithm with the greedy algorithm over a wide range of parameter values. In particular, in Fig. 2, we exhibit the time-averaged system cost vs. the number of participating DS units under various values of $s_{i, \max }$. For all cases, the proposed algorithm achieves much lower system cost, with cost reduction ranging from $11 \%$ to $80 \%$. When the number of DS units increases, the system cost of both algorithms decreases. For the proposed algorithm, Fig. 2 indicates that 150 DS units are enough for the considered power balancing service, while for the greedy algorithm, more DS units are needed to further cut down the system cost. Furthermore, when $s_{i, \max }$ increases, as opposed to the greedy algorithm which cannot benefit from the increased energy range, the proposed algorithm exhibits performance improvement in general.

In Fig. 3, we consider four different values of $\Delta t$ and display the normalized (over $\Delta t$ ) time-averaged system cost vs. the ratio $g_{\max } / \sum_{i=1}^{150} r_{i, \max }$ for different $g_{\max }$ values. For the proposed algorithm, the system cost grows with $\Delta t$ and $g_{\max }$. We observe that when the energy imbalance amount is low, the system cost achieved by the proposed algorithm increases at a much lower rate than that of the greedy algorithm. When the energy imbalance amount is high, even though the system cost achieved by both algorithms increases at nearly the same rate due to saturation of the DS capacity, the proposed algorithm still substantially outperforms the greedy algorithm.

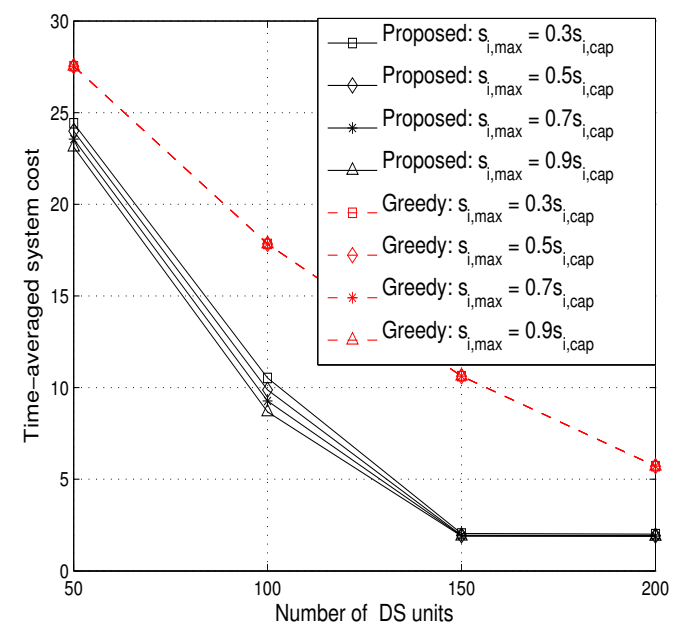

Fig. 2. Time-averaged system cost vs. number of DS units under various $s_{i, \max }$.

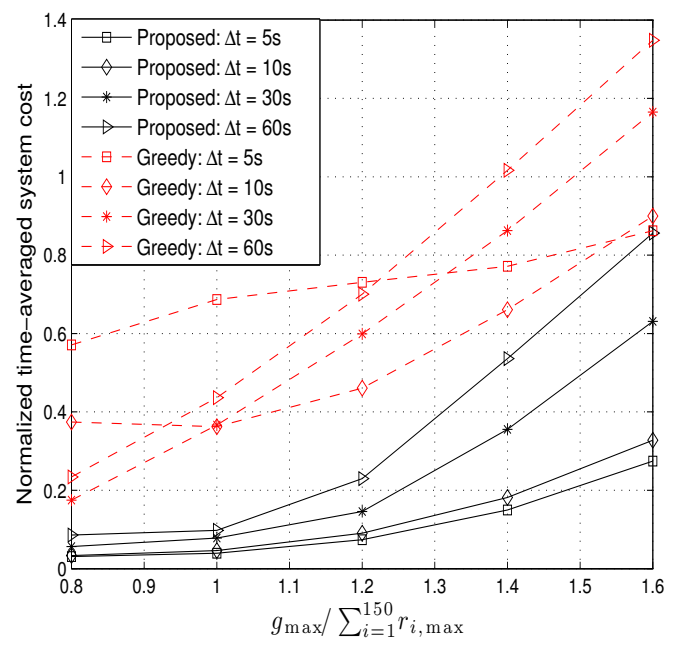

Fig. 3. Normalized time-averaged system cost vs. $g_{\max } / \sum_{i=1}^{150} r_{i, \max }$ under various $\Delta t$.

\section{EXTENSION TO DYNAMIC DS UNITS}

We have so far focused on static DS units that are always connected to the aggregator when providing power balancing service. The case that involves dynamic DS units, i.e., where DS units can leave and rejoin the system such as batteries equipped in electric vehicles, is much more complicated. This is because a returning DS unit may have an energy state different from when it left, and this discrepancy complicates the charging/discharging scheduling to ensure the boundedness of the energy state over multiple time instances.

To overcome this difficulty, we need to reinitialize the virtual queue defined in (14) every time a DS unit rejoins the system, to a properly shifted version of its energy state. The details of this technique can be found in our previous study on employing dynamic electric vehicles in power grids [24]. Similar to the extension from the static case in [23] to the dynamic case in [24], with some mild assumptions, our current analysis can be extended to accommodate dynamic DS units 
and reach similar conclusions as those presented in Section VI.

\section{CONClusion And Future Work}

We have considered a comprehensive aggregator-DS system model to provide real-time power balancing service to a power grid. To minimize the long-term system cost, we have developed a real-time distributed algorithm, by which the statistics of the system is not required and each DS unit can determine its own charging and discharging amounts. The algorithm provably converges quickly and asymptotically achieves the optimal performance as the DS capacity increases. Also, a novel cost cushion parameter has been introduced that tunes the trade-off between system performance and convergence speed. In simulations, we have compared the proposed algorithm with a greedy algorithm over a wide range of parameter values, and demonstrated that the algorithm can offer substantial performance gains.

In this paper the DS units are assumed to exclusively provide real-time power balancing service when they participate in the aggregator-DS system. In particular, their own charging needs (e.g., charging the battery to a certain level before a deadline) are not considered, except that the energy state is ensured to be within a preferred range. In a more general scenario, the DS units, e.g., batteries in electric vehicles, may need to conduct self charging while providing power balancing service. The challenging problem of jointly optimizing self charging and power balancing remains open and is left for future research.

\section{APPENDIX A \\ PROOF OF PROPOSITION 1}

Based on the definition of $L\left(\boldsymbol{\Theta}_{t}\right)$, we have

$L\left(\boldsymbol{\Theta}_{t+1}\right)-L\left(\boldsymbol{\Theta}_{t}\right)=\frac{1}{2} \sum_{i=1}^{N}\left(J_{i, t+1}^{2}-J_{i, t}^{2}+K_{i, t+1}^{2}-K_{i, t}^{2}\right)$.

Using the fact that for any $q \geq 0, b \geq 0$, and $a \geq 0$, there is $(\max \{q-b, 0\}+a)^{2} \leq q^{2}+a^{2}+b^{2}+2 q(a-b)$, we can upper bound $J_{i, t+1}^{2}-J_{i, t}^{2}$ as follows:

$$
\begin{aligned}
J_{i, t+1}^{2}-J_{i, t}^{2} & \leq \hat{l}_{i, u}^{2}+\left(\max \left\{D_{i, c}\left(r_{i, \max }\right), D_{i, d}\left(r_{i, \max }\right)\right\}+a_{i}\right)^{2} \\
& +2 J_{i, t}\left[\mathbf{1}_{s, t} D_{i, c}\left(x_{i, t}\right)+\mathbf{1}_{d, t} D_{i, d}\left(y_{i, t}\right)-l_{i, u}\right] .
\end{aligned}
$$

By the update equation of $K_{i, t}$ in (14), $K_{i, t+1}^{2}-K_{i, t}^{2}$ can be upper bounded by

$$
K_{i, t+1}^{2}-K_{i, t}^{2} \leq 2 K_{i, t} b_{i, t}+r_{i, \max }^{2} .
$$

Imposing the upper bounds (26) (27) on the right hand side of (25), taking conditional expectation on both sides, and then adding the term $V \mathbb{E}\left[w_{t} \mid \boldsymbol{\Theta}_{t}\right]$ give the upper bound of the driftplus-cost function in Proposition 1.

\section{APPENDIX B}

PROOF OF LEMMA 2

The gradient of $G_{t}(\lambda)$ is $G_{t}^{\prime}(\lambda)=g_{t}-\sum_{i=1}^{N} x_{i, t}(\lambda)-$ $q_{t}(\lambda)$, with $x_{i, t}(\lambda) \triangleq\left[\left(D_{i, c}\right)^{\prime-1}\left(\frac{V p_{m, t}+\lambda-K_{i, t} \eta_{i, c}}{J_{i, t}}\right)\right]_{0}^{r_{i, m a x}}$ and $q_{t}(\lambda) \triangleq\left[\left(C_{s}^{\prime}\right)^{-1}\left(\frac{\lambda}{V}\right)\right]_{0}^{g_{t}}$. The second derivative of $G_{t}(\lambda)$ when it exists is

$$
G_{t}^{\prime \prime}(\lambda)=-\sum_{i=1}^{N} x_{i, t}^{\prime}(\lambda)-q_{t}^{\prime}(\lambda)
$$

where $x_{i, t}^{\prime}(\lambda)$ when it exists is

$x_{i, t}^{\prime}(\lambda)= \begin{cases}\frac{1}{J_{i, t} D_{i, c}^{\prime \prime}\left(x_{i, t}(\lambda)\right)}, & D_{i, c}^{\prime}(0) \leq \frac{V p_{m, t}+\lambda-K_{i, t} \eta_{i, c}}{J_{i, t}} \leq D_{i, c}^{\prime}\left(r_{i, \max }\right) \\ 0, & \text { otherwise, }\end{cases}$ and $q_{t}^{\prime}(\lambda)$ when it exists is

$$
q_{t}^{\prime}(\lambda)= \begin{cases}\frac{1}{V C_{s}^{\prime \prime}\left(q_{t}(\lambda)\right)}, & C_{s}^{\prime}(0) \leq \frac{\lambda}{V} \leq C_{s}^{\prime}\left(g_{t}\right) \\ 0, & \text { otherwise. }\end{cases}
$$

Assume that $\lambda_{1}<\lambda_{2}$. Applying the mean value theorem to $G_{t}^{\prime}(\cdot)$, we have

$$
G_{t}^{\prime}\left(\lambda_{1}\right)-G_{t}^{\prime}\left(\lambda_{2}\right)=G_{t}^{\prime \prime}(\epsilon)\left(\lambda_{1}-\lambda_{2}\right),
$$

where $\epsilon \in\left(\lambda_{1}, \lambda_{2}\right)$. Using (28) in (29), there is

$$
\begin{aligned}
& \left|G_{t}^{\prime}\left(\lambda_{1}\right)-G_{t}^{\prime}\left(\lambda_{2}\right)\right| \\
= & \left|G_{t}^{\prime \prime}(\epsilon)\right|\left|\lambda_{1}-\lambda_{2}\right| \\
= & \left(\sum_{i=1}^{N} x_{i, t}^{\prime}(\epsilon)+q_{t}^{\prime}(\epsilon)\right)\left|\lambda_{1}-\lambda_{2}\right| \\
\leq & (N+1) \max \left\{\frac{1}{a_{1} d_{1, l}}, \cdots, \frac{1}{a_{N} d_{N, l}}, \frac{1}{V c_{l}}\right\}\left|\lambda_{1}-\lambda_{2}\right|,
\end{aligned}
$$

where the conditions $\mathbf{C 1}$ and $\mathbf{C 2}$ as well as the fact that $J_{i, t} \geq$ $a_{i}$ are used to derive the last inequality. When the second derivative of $G_{t}(\lambda)$ does not exist, we can replace the gradient in (29) by subgradient and the result still holds [35].

\section{APPENDIX C PROOF OF THEOREM 1}

From Theorem 4.4 of [15], we have the following conclusion: if in Algorithm 1 the step size $\mu=\mu_{0}=1 / \rho$, then the generated sequence $\left\{\lambda^{k}\right\}$ converges to the optimum $\lambda_{t}^{*}$, and for any $k \geq 1$,

$$
G_{t}\left(\lambda_{t}^{*}\right)-G_{t}\left(\lambda^{k}\right) \leq \frac{2 \rho\left|\lambda^{0}-\lambda_{t}^{*}\right|^{2}}{(k+1)^{2}}
$$

where $\lambda^{0}$ is the initial value of $\lambda$.

By the fact that if a function is Lipschitz continuous for the Lipschitz constant $\rho$, then the function is also Lipschitz continuous for all finite constant $\rho^{\prime} \geq \rho$, we can easily obtain Theorem 1 using Theorem 4.4 of [15] for all $\mu \in\left(0, \mu_{0}\right]$. 
APPENDIX D

PROOF OF PROPOSITION 2

By the Karush-Kuhn-Tucker (KKT) conditions, at the optimal point of P2(a'), the following optimality conditions hold

$\begin{cases}\frac{J_{i, t}}{V} D_{i, c}^{\prime}(0)-p_{m, t}+\frac{K_{i, t} \eta_{i, c}}{V}-\frac{\lambda_{t}^{*}}{V} \geq 0, & \text { if } x_{i, t}^{*}=0 \\ \frac{J_{i, t}}{V} D_{i, c}^{\prime}\left(x_{i, t}^{*}\right)-p_{m, t}+\frac{K_{i, t} \eta_{i, c}}{V}-\frac{\lambda_{t}^{*}}{V}=0, & \text { if } 0<x_{i, t}^{*}<r_{i, \max } \\ \frac{J_{i, t}}{V} D_{i, c}^{\prime}\left(r_{i, \max }\right)-p_{m, t}+\frac{K_{i, t} \eta_{i, c}}{V}-\frac{\lambda_{t}^{*}}{V} \leq 0, & \text { if } x_{i, t}^{*}=r_{i, \max } .\end{cases}$

Suppose that under the condition of Proposition 2, we have the contrary, i.e., $\frac{\lambda_{t}^{*}}{V} \leq \min _{1 \leq i \leq N}\left\{\frac{J_{i, t}}{V} D_{i, c}^{\prime}(0)-p_{m, t}+\frac{K_{i, t} \eta_{i, c}}{V}\right\}$, and thus $\mathbf{x}_{t}^{*}=\mathbf{0}$ from (30). Then we will show that we can find another solution with all elements zero except the $j$-th element equal to $\epsilon$ resulting in a strictly smaller objective value, which is a contradiction. Using the objective function of P2(a'), this is equivalent to showing

$$
\frac{J_{j, t}}{V} D_{j, c}(\epsilon)-p_{m, t} \epsilon+\frac{K_{j, t} \eta_{j, c}}{V} \epsilon<C_{s}\left(g_{t}\right)-C_{s}\left(g_{t}-\epsilon\right) .
$$

By the mean value theorem, from the left hand side of (31),

$$
\begin{aligned}
& \frac{J_{j, t}}{V} D_{j, c}(\epsilon)-p_{m, t} \epsilon+\frac{K_{j, t} \eta_{j, c}}{V} \epsilon \\
& =\epsilon\left[\frac{J_{j, t}}{V} D_{j, c}^{\prime}\left(\delta_{1}\right)-p_{m, t}+\frac{K_{j, t} \eta_{j, c}}{V}\right]
\end{aligned}
$$

where $0<\delta_{1}<\epsilon$; from the right hand side of (31), we have

$$
C_{s}\left(g_{t}\right)-C_{s}\left(g_{t}-\epsilon\right)=\epsilon C_{s}^{\prime}\left(\delta_{2}\right)
$$

where $g_{t}-\epsilon<\delta_{2}<g_{t}$. Using (32) and (33), (31) is equivalent to

$$
\frac{J_{j, t}}{V} D_{j, c}^{\prime}\left(\delta_{1}\right)-p_{m, t}+\frac{K_{j, t} \eta_{j, c}}{V}<C_{s}^{\prime}\left(\delta_{2}\right) .
$$

(34) is true since we have $C_{s}^{\prime}\left(\delta_{2}\right)>C_{s}^{\prime}\left(\delta_{1}\right)>\frac{J_{j, t}}{V} D_{j, c}^{\prime}\left(\delta_{1}\right)-$ $p_{m, t}+\frac{K_{j, t} \eta_{j, c}}{V}$, where the first inequality is due to $g_{t} \gg \epsilon$ and $C_{s}^{\prime \prime}(\cdot)>0$, and the second inequality is based on the condition of Proposition 2.

\section{APPENDiX E \\ Proof of LEMMA 3}

1) Consider $g_{t}>0$. Suppose that when $K_{i, t}>$ $V \frac{\left(p_{m, \max }+c_{\max }\right)}{\eta_{i, c}}$, the optimal solution under the proposed algorithm is $\tilde{\mathbf{x}}_{t}$ with $\tilde{x}_{i, t}>0$. Then we show that we can find another solution $\hat{\mathbf{x}}_{t}$ with $\hat{x}_{j, t}=\tilde{x}_{j, t}, \forall j \neq i$, and $\hat{x}_{i, t}=0$, resulting in a strictly smaller objective value, which is a contradiction.

Using the objective function of P2(a), this is equivalent to showing that

$$
\begin{aligned}
& {\left[\sum_{j=1}^{N} J_{j, t} D_{j, c}\left(\tilde{x}_{j, t}\right)-V p_{m, t} \tilde{x}_{j, t}+K_{j, t} \eta_{j, c} \tilde{x}_{j, t}\right]} \\
& +V C_{s}\left(g_{t}-\sum_{j=1}^{N} \tilde{x}_{j, t}\right) \\
& >\left[\sum_{j \neq i}^{N} J_{j, t} D_{j, c}\left(\tilde{x}_{j, t}\right)-V p_{m, t} \tilde{x}_{j, t}+K_{j, t} \eta_{j, c} \tilde{x}_{j, t}\right]
\end{aligned}
$$

$$
+V C_{s}\left(g_{t}-\sum_{j=1}^{N} \tilde{x}_{j, t}+\tilde{x}_{i, t}\right)
$$

which is equivalent to

$$
\begin{aligned}
& J_{i, t} D_{i, c}\left(\tilde{x}_{i, t}\right)-V p_{m, t} \tilde{x}_{i, t}+K_{i, t} \eta_{i, c} \tilde{x}_{i, t} \\
& >V\left[C_{s}\left(g_{t}-\sum_{j=1}^{N} \tilde{x}_{j, t}+\tilde{x}_{i, t}\right)-C_{s}\left(g_{t}-\sum_{j=1}^{N} \tilde{x}_{j, t}\right)\right] \\
& =V \tilde{x}_{i, t} C_{s}^{\prime}(\epsilon)
\end{aligned}
$$

where (35) is derived by the mean value theorem with $\epsilon \in$ $\left(g_{t}-\sum_{j=1}^{N} \tilde{x}_{j, t}, g_{t}-\sum_{j=1}^{N} \tilde{x}_{j, t}+\tilde{x}_{i, t}\right)$. Since $J_{i, t} D_{i, c}\left(\tilde{x}_{i, t}\right) \geq$ 0 , from (35), it suffices to show that

$$
\left[K_{i, t} \eta_{i, c}-V p_{m, t}-V C_{s}^{\prime}(\epsilon)\right] \tilde{x}_{i, t}>0 .
$$

Since $\tilde{x}_{i, t}>0, p_{m, t} \leq p_{m, \max }$, and $C_{s}^{\prime}(\epsilon) \leq c_{\max }$, (36) is true by using the condition that $K_{i, t}>\frac{V\left(c_{\max }+p_{\max }\right)}{\eta_{i, c}}$.

2) Consider $g_{t}<0$. Suppose that when $K_{i, t}<V\left(p_{m, \min }-\right.$ $\left.\frac{c_{\max }}{\eta_{i, d}}\right)$, the optimal solution under the proposed algorithm is $\tilde{\mathbf{y}}_{t}$ with $\tilde{y}_{i, t}>0$. Then there is a contradiction since we can construct another solution $\hat{\mathbf{y}}_{t}$ with $\hat{y}_{j, t}=\tilde{y}_{j, t}, \forall j \neq i$, and $\hat{y}_{i, t}=0$, which results in a strictly smaller objective value. The proof is similar to that in 1) and is omitted here.

\section{APPENDIX F \\ PRoOF OF LEMMA 4}

The proof proceeds by induction over time $t$. The base case trivially holds. For the inductive step, first consider the upper bound. Assume that $K_{i, t} \leq s_{i, \max }-\beta_{i}$ holds at time slot $t$. Consider the following two cases.

Case 1: $V \frac{\left(p_{m, \max }+c_{\max }\right)}{\eta_{i, c}}<K_{i, t} \leq s_{i, \max }-\beta_{i}$. (It is easy to check that $V \frac{\left(p_{m, \max }+c_{\max }\right)}{\eta_{i, c}}<s_{i, \max }-\beta_{i}$ since $V \leq V_{\max }$.) For $g_{t}>0$, from Lemma $3, x_{i, t}^{*}=0$; therefore, based on the update equation (14), there is $K_{i, t+1}=K_{i, t} \leq s_{i, \max }-\beta_{i}$. For $g_{t}<0$, we have $K_{i, t+1}=K_{i, t}-\eta_{i, d} y_{i, t} \leq K_{i, t} \leq s_{i, \max }-\beta_{i}$.

Case 2: $K_{i, t} \leq V \frac{\left(p_{m, \max }+c_{\max }\right)}{\eta_{i, c}}$. From (14), $K_{i, t+1} \leq$ $V \frac{\left(p_{m, \max }+c_{\max }\right)}{\eta_{i, c}}+\eta_{i, c} r_{i, \max } \leq s_{i, \max }-\beta_{i}$, where the last inequality holds since $V \leq V_{\max }$.

We now consider the lower bound. Assume that $K_{i, t} \geq$ $s_{i, \min }-\beta_{i}$ holds at time slot $t$. Consider the following two cases.

Case $1^{\prime}: s_{i, \min }-\beta_{i} \leq K_{i, t}<V\left(p_{m, \min }-\frac{c_{\max }}{\eta_{i, d}}\right)$. (It is easy to check that $s_{i, \min }-\beta_{i}<V\left(p_{m, \min }-\frac{c_{\max }}{\eta_{i, d}}\right)$ since $r_{i, \max }>0$.) For $g_{t}<0$, from Lemma $3, y_{i, t}^{*}=0$; therefore, $K_{i, t+1}=K_{i, t} \geq s_{i, \min }-\beta_{i}$. For $g_{t}>0$, from (14), $K_{i, t+1}=$ $K_{i, t}+\eta_{i, c} x_{i, t} \geq K_{i, t} \geq s_{i, \min }-\beta_{i}$.

Case $2^{\prime}: K_{i, t} \geq V\left(p_{m, \min }-\frac{c_{\max }}{\eta_{i, d}}\right)$. From (14), $K_{i, t+1} \geq$ $V\left(p_{m, \min }-\frac{c_{\max }}{\eta_{i, d}}\right)-\eta_{i, d} r_{i, \max } \geq s_{i, \min }-\beta_{i}$, where the last inequality holds based on the definition of $\beta_{i}$.

\section{APPENDIX G \\ Proof of Theorem 2}

Consider the problem $\mathbf{P 2}$, and denote the optimal long-term system cost for $\mathbf{P} 2$ as $\hat{f}$. We first prove the following lemma, which will be used later. 
Lemma 6: For $\mathbf{P 2}$, there exists a stationary randomized regulation allocation solution $\left(\mathbf{x}_{t}^{s}, \mathbf{y}_{t}^{s}\right)$ that only depends on the system state $A_{t}$, and at the same time satisfies the following conditions:

$$
\begin{aligned}
& \mathbb{E}\left[w_{t}^{s}\right] \leq \hat{f}, \\
& \mathbb{E}\left[\mathbf{1}_{s, t} D_{i, c}\left(x_{i, t}^{s}\right)+\mathbf{1}_{d, t} D_{i, d}\left(y_{i, t}^{s}\right)-l_{i, u}\right] \leq 0, \forall i, \\
& \mathbb{E}\left[b_{i, t}^{s}\right]=0, \forall i,
\end{aligned}
$$

where the expectations are taken over the randomness of the system and the randomness of $\left(\mathbf{x}_{t}^{s}, \mathbf{y}_{t}^{s}\right)$.

Proof: The claims above can be derived from Theorem 4.5 in [13]. In particular, that theorem implies that the sufficient conditions for the existence of a stationary and randomized algorithm as described in Lemma 6 are as follows: first, the system state $A_{t}$ is stationary; second, the system satisfies the boundedness assumptions and the law of large numbers; and third, $\mathbf{P 2}$ is feasible. It is easy to check that $\mathbf{P 2}$ is feasible. In addition, since we have assumed that $A_{t}$ is i.i.d. and the variables $g_{t}, x_{i, t}, y_{i, t}$, and $p_{m, t}$ are bounded, these sufficient conditions are all met in our problem. Therefore, the conclusion in Lemma 6 holds.

Since the proposed algorithm minimizes the upper bound of the drift-plus-cost function at each time, plugging $\left(\mathbf{x}_{t}^{s}, \mathbf{y}_{t}^{s}\right)$ into the right hand side of (17) and using (37), (38), and (39) yields

$$
\Delta\left(\boldsymbol{\Theta}_{t}\right)+V \mathbb{E}\left[w_{t} \mid \boldsymbol{\Theta}_{t}\right] \leq B+V \hat{f} \leq B+V f^{\text {opt }},
$$

where the last inequality holds since $\mathbf{P 2}$ is a relaxed problem of $\mathbf{P 1}$ hence having a smaller objective value.

We first prove the result in 2). Taking expectations over $\boldsymbol{\Theta}_{t}$ on both sides of (40) and summing over $t \in\{0, \cdots, T-1\}$ gives

$$
\mathbb{E}\left[L\left(\boldsymbol{\Theta}_{T}\right)\right]-\mathbb{E}\left[L\left(\boldsymbol{\Theta}_{0}\right)\right]+V \sum_{t=0}^{T-1} \mathbb{E}\left[w_{t}\right] \leq\left(B+V f^{\text {opt }}\right) T .
$$

After some arrangement, from (41), there is

$$
\frac{1}{T} \sum_{t=0}^{T-1} \mathbb{E}\left[w_{t}\right] \leq \frac{B+V f^{\mathrm{opt}}}{V}+\frac{\mathbb{E}\left[L\left(\boldsymbol{\Theta}_{0}\right)\right]}{T V} .
$$

Taking $T \rightarrow \infty$ gives $\lim _{T \rightarrow \infty} \frac{1}{T} \sum_{t=0}^{T-1} \mathbb{E}\left[w_{t}\right] \leq \frac{B}{V}+$ $f^{\text {opt }}, V \in\left(0, V_{\text {max }}\right]$, which is exactly the conclusion in 2$)$.

We now prove the result in 1). From (41), we have

$$
\mathbb{E}\left[L\left(\boldsymbol{\Theta}_{T}\right)\right] \leq \mathbb{E}\left[L\left(\boldsymbol{\Theta}_{0}\right)\right]+\left[B+V\left(f^{\text {opt }}-f_{\text {min }}\right)\right] T,
$$

where $f_{\min } \triangleq-p_{m, \max } \sum_{i=1}^{N} r_{i, \max }$. Using the fact that $\mathbb{E}\left[J_{i, T}\right] \leq \sqrt{\mathbb{E}\left[J_{i, T}^{2}\right]} \leq \sqrt{2 \mathbb{E}\left[L\left(\boldsymbol{\Theta}_{T}\right)\right]}$, from (43) we get

$$
\mathbb{E}\left[J_{i, T}\right] \leq \sqrt{2\left(\mathbb{E}\left[L\left(\boldsymbol{\Theta}_{0}\right)\right]+\left[B+V\left(f^{\text {opt }}-f_{\min }\right)\right] T\right)} .
$$

Dividing both sides of (44) by $T$ and taking limits gives $\lim _{T \rightarrow \infty} \frac{\mathbb{E}\left[J_{i, T}\right]}{T}=0$. Hence, by Lemma 1, the virtual queue $J_{i, t}$ is mean rate stable and constraint (8) holds. Using a similar argument, we can show that the virtual queue $K_{i, t}$ is mean rate stable and constraint (9) holds. Also, since we have proven in Lemma 5 that the energy state is bounded within the preferred range, $\left\{\mathbf{x}_{t}^{*}, \mathbf{y}_{t}^{*}\right\}$ is feasible for $\mathbf{P 1}$.

\section{REFERENCES}

[1] A. Meier, Electric Power Systems: A Conceptual Introduction. WileyIEEE Press, 2006.

[2] B. Kirby, "Frequency regulation basics and trends," U.S. Dept. Energy, Tech. Rep., 2005.

[3] European Commission, "Package of implementation measures for the EU's objectives on climate change and renewable energy for 2020," 2008. [Online]. Available: http://ec.europa.eu/clima/policies/package/ docs/sec_2008_85_ia_en.pdf

[4] Senator energy, utilities and communications commitee, "Senate Bill X 1-2," 2011. [Online]. Available: http://www.leginfo.ca.gov/pub/11-12/ bill/sen/sb_0001-0050/sbx1_2_cfa_20110214_141136_sen_comm.html

[5] B. Narayanaswamy, V. Garg and T. Jayram, "Online optimization for the smart (micro) grid," in ACM e-Energy, May 2012.

[6] L. Lu, J. Tu, C. Chau, M. Chen, and X. Lin, "Online energy generation scheduling for microgrids with intermittent energy sources and cogeneration," in Proc. ACM Sigmetrics, Jun. 2013.

[7] T. Chang, M. Alizadeh, and A. Scaglione, "Real-time power balancing via decentralized coordinated home energy scheduling," IEEE Trans. Smart Grid, vol. 4, pp. 1490-1504, Sep. 2013.

[8] G. Jóos, B. Ooi, D. McGillis, F. Galiana, and R. Marceau, "The potential of distributed generation to provide ancillary services," in Proc. IEEE Power Eng. Soc. Summer Meeting, Jan. 2000.

[9] W. Kempton, V. Udo, K. Huber, K. Komara, S. Letendre, S. Baker, D. Brunner, and N. Pearre, "A test of vehicle-to-grid (V2G) for energy storage and frequency regulation in the PJM system," Tech. Rep., Nov. 2008. [Online]. Available: http://www.udel.edu/V2G/resources/ test-v2g-in-pjm-jan09.pdf

[10] "Electric drive sales dashboard." [Online]. Available: http://electricdrive. org/index.php?ht=d/sp/i/20952/pid/20952

[11] J. John , "SolarCity and Tesla: a utility's worst nightmare?" Mar. 2014. [Online]. Available: http://www.greentechmedia.com/articles/read/ SolarCitys-Networked-Grid-Ready-Energy-Storage-Fleet

[12] D. Hill, A. Agarwal, and F. Ayello, "Fleet operator risks for using fleets for V2G regulation," ENERG POLICY, vol. 41, pp. 221-231, Feb. 2012.

[13] M. Neely, Stochastic Network Optimization with Application to Communication and Queueing Systems. Morgan \& Claypool, 2010.

[14] S. Boyd and L. Vandenberghe, Convex Optimization. Cambridge University Press, 2004.

[15] A. Beck and M. Teboulle, "A fast iterative shrinkage-thresholding algorithm for linear inverse problems," SIAM J. Imaging Sci., vol. 2, pp. 183-202, 2009.

[16] Y. Huang, S. Mao, and R. Nelms, "Adaptive electricity scheduling in microgrids." [Online]. Available: arXiv:1301.0528v1

[17] Y. Guo, M. Pan, Y. Fang, and P. Khargonekar, "Decentralized coordination of energy utilization for residential households in the smart grid," IEEE Trans. Smart Grid, vol. 4, pp. 1341-1350, Sep. 2013.

[18] J. Garzas, A. Armada, and G. Granados, "Fair design of plug-in electric vehicles aggregator for $\mathrm{V} 2 \mathrm{G}$ regulation," IEEE Trans. Veh. Technol., vol. 61, pp. 3406-3419, Oct. 2012.

[19] E. Sortomme and M. Sharkawi, "Optimal scheduling of vehicle-to-grid energy and ancillary services," IEEE Trans. Smart Grid, vol. 3, pp. 351359, Mar. 2012.

[20] S. Han, S. Han, and K. Sezaki, "Optimal control of the plug-in electric vehicles for $\mathrm{V} 2 \mathrm{G}$ frequency regulation using quadratic programming," in Proc. IEEE ISGT, Jan. 2011.

[21] - "Development of an optimal vehicle-to-grid aggregator for frequency regulation," IEEE Trans. Smart Grid, vol. 1, pp. 65-72, Jun. 2010.

[22] W. Shi and V. Wong, "Real-time vehicle-to-grid control algorithm under price uncertainty," in Proc. IEEE SmartGridComm, Oct. 2011.

[23] S. Sun, M. Dong, and B. Liang, "Real-time welfare-maximizing regulation allocation in aggregator-EVs systems," in Proc. IEEE INFOCOM Workshop on CCSES, Apr. 2013.

[24] — , "Real-time welfare-maximizing regulation allocation in dynamic aggregator-EVs system," IEEE Trans. Smart Grid, vol. 5, May 2014.

[25] C. Wu, H. Rad, and J. Huang, "Vehicle-to-aggregator interaction game," IEEE Trans. Smart Grid, vol. 3, pp. 434-441, Mar. 2012.

[26] B. Gharesifard, T. Başar, and A. Domínguez-García, "Price-based distributed control for networked plug-in electric vehicles," in Proc. ACC, Jun. 2013.

[27] S. Sun, M. Dong, and B. Liang, "Distributed regulation allocation with aggregator coordinated electric vehicles," in Proc. IEEE SmartGridComm., Oct. 2013. 
[28] S. Han, S. Han, and K. Sezaki, "Economic assessment on V2G frequency regulation regarding the battery degradation," in Proc. IEEE ISGT, Jan. 2012.

[29] P. Ramadass, B. Haran, R. White, and B. Popov, "Performance study of commercial LiCoO2 and spinel-based Li-ion cells," J. Power Sources, vol. 111, pp. 210-220, Apr. 2002.

[30] E. Bitar, A. Giani, R. Rajagopal, D. Varagnolo, P. Khargonekar, K. Poolla, and P. Varaiya, "Optimal contracts for wind power producers in electricity markets," in Proc. IEEE CDC, Dec. 2010.

[31] D. Bertsekas, Dynamic Programming and Optimal Control. Athena Scientific, 2005

[32] R. Urgaonkar, B. Urgaonkar, M. Neely, and A. Sivasubramaniam, "Optimal power cost management using stored energy in data centers," in Proc. ACM SIGMETRICS, 2011.

[33] D. Bertsekas, Nonlinear Programming. Athena Scientific, 1999.

[34] "Electricity prices in ontario." [Online]. Available: http://www. ontarioenergyboard.ca/OEB/Consumers/Electricity/Electricity+Prices

[35] S. Low and D. Lapsley, "Optimization flow control - I: basic algorithm and convergence," IEEE/ACM Trans. Netw., vol. 7, pp. 861-874, Dec. 1999.

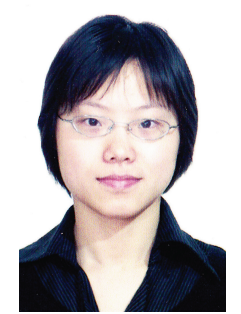

Sun Sun (S'11) received the B.S. degree in Electrical Engineering and Automation from Tongji University, Shanghai, China, in 2005. From 2006 to 2008, she was a software engineer in the Department of GSM Base Transceiver Station of Huawei Technologies Co. Ltd.. She received the M.Sc. degree in Electrical and Computer Engineering from University of Alberta, Edmonton, Canada, in 2011. Now, she is pursuing her Ph.D. degree in the Department of Electrical and Computer Engineering of University of Toronto, Toronto, Canada. She is interested in the areas of stochastic optimization, distributed control, and network resource management. Her current research lies in applying communication, information, and control technologies to smart grid, focusing on renewable energy integration, energy storage, and demand response.

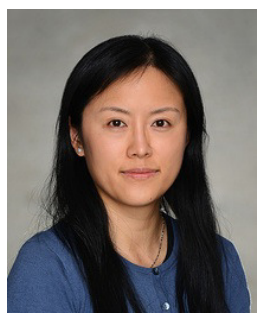

Min Dong (S'00-M'05-SM'09) received the B.Eng. degree from Tsinghua University, Beijing, China, in 1998, and the Ph.D. degree in electrical and computer engineering with minor in applied mathematics from Cornell University, Ithaca, NY, in 2004. From 2004 to 2008, she was with Corporate Research and Development, Qualcomm Inc., San Diego, CA. In 2008, she joined the Department of Electrical, Computer and Software Engineering at University of Ontario Institute of Technology, Ontario, Canada, where she is currently an Associate Professor. She also holds a status-only Associate Professor appointment with the Department of Electrical and Computer Engineering, University of Toronto since 2009. Her research interests are in the areas of statistical signal processing for communication networks, cooperative communications and networking techniques, and stochastic network optimization in dynamic networks and systems.

Dr. Dong received the Early Researcher Award from Ontario Ministry of Research and Innovation in 2012, the Best Paper Award at IEEE ICCC in 2012, and the 2004 IEEE Signal Processing Society Best Paper Award. She was an Associate Editor for the IEEE SIGNAL PROCESSING LETTERS during 2009-2013, and currently serves as an Associate Editor for the IEEE TRANSACTIONS ON SIGNAL PROCESSING. She has been an elected member of IEEE Signal Processing Society Signal Processing for Communications and Networking (SP-COM) Technical Committee since 2013.

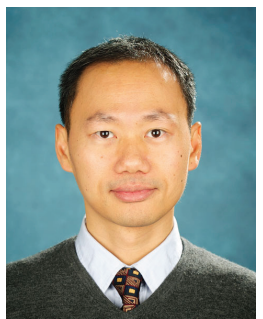

Ben Liang (S'94-M'01-SM'06) received honorssimultaneous B.Sc. (valedictorian) and M.Sc. degrees in Electrical Engineering from Polytechnic University in Brooklyn, New York, in 1997 and the Ph.D. degree in Electrical Engineering with Computer Science minor from Cornell University in Ithaca, New York, in 2001. In the 2001 - 2002 academic year, he was a visiting lecturer and postdoctoral research associate at Cornell University. $\mathrm{He}$ joined the Department of Electrical and Computer Engineering at the University of Toronto in 2002 , where he is now a Professor. His current research interests are in mobile communications and networked systems. He has served as an editor for the IEEE Transactions on Wireless Communications and an associate editor for the Wiley Security and Communication Networks journal, in addition to regularly serving on the organizational or technical committee of a number of conferences. He is a senior member of IEEE and a member of ACM and Tau Beta Pi. 